\title{
Global Well-posedness for the Three Dimensional Simplified Inertial Ericksen-Leslie Systems Near Equilibrium
}

\author{
Yuan Cai * Wei Wang ${ }^{\dagger}$
}

\begin{abstract}
We study a simplified inertial Ericksen-Leslie system for the nematic liquid crystal flow, which can be viewed as a system coupling Navier-Stokes equations and wave map equations. We prove the global existence of classical solution with initial data near equilibrium.
\end{abstract}

\section{Introduction}

The Ericksen-Leslie system is a hydrodynamical theory for nematic liquid crystals which was established by Ericksen 4 and Leslie [28 in 1960's. It has been successful to model various dynamical behavior for nematic liquid crystals. In this paper, we consider the simplified Ericksen-Leslie system 28. with the inertial term:

$$
\left\{\begin{array}{l}
\partial_{t} v+v \cdot \nabla v+\nabla p=\mu \Delta v-\nabla \cdot(\nabla d \otimes \nabla d) \\
\sigma_{0} D_{t}^{2} d+\sigma_{1} D_{t} d-\Delta d=\left(|\nabla d|^{2}-\sigma_{0}\left|D_{t} d\right|^{2}\right) d \\
\nabla \cdot v=0
\end{array}\right.
$$

on $\mathbb{R}^{n} \times \mathbb{R}^{+}(n \geq 2)$. Here $v(x, t) \in \mathbb{R}^{n}$ is the average velocity of liquid crystal molecules, $p$ is the pressure, and $d(x, t) \in \mathbb{S}^{2}$ is the directional field representing the orientation alignment of liquid crystal molecules. $D_{t}=\left(\partial_{t}+v \cdot \nabla\right)$ denotes the material derivative. The first two equations in (1.1) represent the conservation of momentum and angular momentum respectively. The third equation is the incompressibility condition for the velocity. The term $\left(|\nabla d|^{2}-\sigma_{0}\left|D_{t} d\right|^{2}\right) d$ on the right hand side of (1.1) 2 plays the role of Lagrangian multiplier for the constraint $|d|=1$. The term $\sigma_{0} D_{t}^{2} d$ is called the inertial term, while $\sigma_{1} D_{t} d$ is called the damping term. If $\sigma_{0}=0, \sigma_{1}>0$, then $(1.1)_{2}$ is called the simplified (non-inertial) Ericksen-Leslie system which is a parabolic type equation and has been widely studied in literatures since the work of Lin etc. [31, 33, 34, 35. If $\sigma_{0}>0, \sigma_{1}=0$, then (1.1) becomes a hyperbolic one. We remark that the system considered here neglects complicated Leslie's stress terms in the momentum equation and corresponding co-rotational and stretching terms in the angular momentum equation, however, the inertial term is kept. We refer to [5, 28, or [10] for its mathematical derivation and the full form.

There have been many works on the non-inertial Ericksen-Leslie system. For the simplified system without Leslie's stress, Lin-Lin-Wang [32] and Hong [6] established the existence of global weak solutions in $\mathbb{R}^{2}$, see also Lin-Wang [36], Xu-Zhang [59, Hong-Xin [7] and Lei-Li-Zhang [25] for related results in the two dimensional case. Recently, Lin-Wang [37. proved the global existence of weak solution for dimension three when the initial alignments $d_{0}$ locates in the upper half sphere. We also refer to [30, 9] for global existence of strong solution with small data for dimension three. For the full non-inertial system with Leslie's stress, Wang-Zhang-Zhang [58] proved the local existence for general data and global existence of smooth solutions for data near equilibrium under optimal constraints

\footnotetext{
*Department of Mathematics, The Hong Kong University of Science and Technology, Clear Water Bay, Kowloon, Hong Kong. Email: maycai@ust.hk

†School of Mathematical Sciences, Zhejiang University, Hangzhou 310027, P. R. China. Email: wangw07@zju.edu.cn
} 
on the Leslie coefficients for three dimensional case. Wang-Wang [56] extended these results to the general Oseen-Frank energy case. For 2D case, the existence of global weak solutions was proved by Wang-Wang [56] and Huang-Lin-Wang [8], while the uniqueness of weak solutions was considered by Wang-Wang-Zhang [57] and Li-Titi-Xin [29].

To the best knowledge of the authors, the results considering the Ericksen-Leslie system with inertial term are very few, especially in space dimensions higher than one. There are some results on the one dimensional problem with general Oseen-Frank energy, in which, however, the fluid coupling is neglected (i. e. $v=0$ ). We refer to $2,60,61,62$ and references therein for examples. For the full inertial Ericksen-Leslie system, Jiang-Luo [10] studied the local wellposedness of classical solutions as well as the global wellposedness when the damping coefficients $\sigma_{1}>0$.

In this work, we study the global wellposedness of classical solutions to the simplified inertial Ericksen-Leslie system (1.1) in three dimensions for the case $\sigma_{1}=0$. We remark that the absence of the term $\sigma_{1} D_{t} d$ makes the system more difficult to study than the case of $\sigma_{1}>0$ which bring an additional damping effect. In general, for quasi-linear wave equations, the solution may blow up in finite time even if the initial data are smooth and small. However, due to the null structure nature of the nonlinear term in $(1.1)_{2}$, one can expect existences of global smooth solutions with small initial data. Actually, if the fluid velocity is ignored, the system is just a equation for wave maps, which the null condition is satisfied and is now well known to have global small solutions.

In addition, we assume $\sigma_{0}=1, \mu=1$ without loss of generality. The main result of this paper is stated as follows (the notations will be explained in Section 2.2):

Theorem 1.1. Let $\left(v_{0}(x), d_{0}(x)-e\right) \in H_{\Lambda}^{\kappa}$ with $\kappa \geq 9$ where $e \in \mathbb{S}^{2}$ is some constant director. Suppose

$$
\left\|\left(v_{0}, d_{0}-e, d_{1}\right)\right\|_{H_{\Lambda}^{\kappa}}^{2} \leq \epsilon .
$$

There exists a positive constant $\epsilon_{0}$ such that, if $\epsilon \leq \epsilon_{0}$, the system (1.1) with initial data

$$
v(x, 0)=v_{0}(x), \quad d(x, 0)=d_{0}(x), \quad \partial_{t} d(x, 0)=d_{1}(x)
$$

has a unique global classical solution such that

$$
E_{\kappa+1}^{d}(t) \leq C_{0} \epsilon\langle t\rangle^{\delta}, \quad E_{\kappa}^{v}+E_{\kappa-1}^{d}(t) \leq C_{0} \epsilon
$$

for some positive $C_{0}>0,0<\delta<\frac{1}{2}$ depending on $\kappa$ and $\epsilon_{0}$ uniformly for all $0 \leq t<\infty$.

Remark 1.1. Following the recent works $[3,24,26$, we can apply the ghost weight energy method [1] to even prove a uniform bound for the highest-order energy. However, as the current estimate is sufficient to obtain the global existences of solutions, we will not pursue this direction in this paper.

Remark 1.2. The regularity index $\kappa$ may be lowered a little bit. However, restricted by vector field method, we can not expect it close to the natural energy space. Hence, we do not aim to find the lowest regularity index here.

Remark 1.3. Inspired by the threshold global regularity on wave maps 42, 43, it might be natural to conjecture that the similar results also hold for the two dimensional inertial liquid crystal. This issue will be addressed in our future work.

We will perform the analysis under Eulerian coordinates, although Lagrangian coordinates may also work. The main difficulty in proving global solutions lies in the contradiction between parabolicity and the hyperbolicity. In another words, the parabolic method and the hyperbolic approach are not compatible. To overcome this essential difficulty, we need use an unified approach working both in parabolic equations and hyperbolic ones. This is the main novelty of this paper.

Considering the wave nature of the equation for $d$, we use the vector field theory and the weighted energy method. However, the system has neither Lorentz invariance nor scaling invariance. We use the weighted $L^{2}$ norm introduced by Klainerman and Sideris [17] to overcome the difficulty from the lack of Lorentz invariance, while the lack of scaling invariance is solved by directly applying the 
scaling operator and dealing with the commutators [11. To close the energy estimate, it is necessary to obtain the subcritical decay for the solution. However, the interaction between velocity filed and the orientation field weakens the dissipative nature of the velocity field and the dispersive effect of the orientation filed, and even worse, it is strengthened by the quasilinear nature of the system. These difficulties make the decay estimate very delicate. In addition, to close the higher-order energy estimate, we need to explore the symmetry structure of the system to deal with the quasilinear terms which may cause loss of derivatives at a first glance. This structure is not obvious in the Eulerian formulation.

Before ending the introduction, we simply review some related results concerning the wave maps which corresponds to the inertial Ericksen-Leslie system (1.1) when velocity is equal zero and $\sigma_{0}=1$, $\sigma_{1}=0$. The Cauchy problem for the wave maps has been extensively studied, especially when space dimension is two, where the scaling is invariant in the natural energy space. In the supercritical case $n \geq 3$, it was observed by Shatah [40] that there exist self-similar blow up solutions of finite energy. In the critical case $\mathrm{n}=2$, Struwe 44] observed that in the equivariant setting, blow up in this dimension must result from a strictly slower than self-similar rescaling of a harmonic sphere of finite energy. Finally, the blow up for this more difficult two-dimensional case was by exhibited by Rodnianski-Sterbenz [39, as well as Krieger-Schlag-Tataru 23]. As for the well-posedness results, the wave map displays a null form structure, which was the essential feature in the subcritical theory of Klainerman-Machedon [12, 13, 14, and Klainerman-Selberg 15, 16. These authors proved strong local well-posedness for data in $H^{s}\left(\mathbb{R}^{n}\right)$ when $s>n / 2$. The important critical theory $s=n / 2$ was begun by Tataru [52, 53]. In a breakthrough work, Tao [45, 46] proved the global regularity for small initial data in $\dot{H}^{\frac{n}{2}}\left(\mathbb{R}^{n}\right) \times \dot{H}^{\frac{n}{2}-1}\left(\mathbb{R}^{n}\right)$ when the target is a sphere by renormalization of the geometric structure. Afterwards-Klainerman-Rodnianski [18, Nahmod-Stephanov-Uhlenbeck [38, Tataru [54, 55], and Krieger [19, 20, 21] considered other cases of targets by using similar methods as in Tao's work. As for large initial data, Sterbenz-Tataru [42, 43] gave the following satisfactory result: If the energy of the initial data is smaller than the energy of any nontrivial harmonic map $\mathbb{R}^{n} \rightarrow M$, then one has the global existence theory. A particular case is the hyperbolic space $\mathbb{H}^{n}$ for which Tao [47, 48, 49, 50, 51] as well as Krieger-Schlag [22, has achieved the same result.

The remaining part of this paper is organized as follows. In the next section, we will introduce the vector fields applied onto the system, ansatz for the method of continuity, and some preliminary estimates. In Section 3, we will estimate of weighted $L^{2}$ norm. Section 4 is devoted to the decay estimate of velocity. In the last section, we give various energy estimates which are crucial steps to prove Theorem 1.

\section{Preliminaries}

\subsection{Application of vector fields}

As stated in the introduction, we will use various vector fields which play a central role in the proofs. To begin with, we rewrite the system (1.1) as

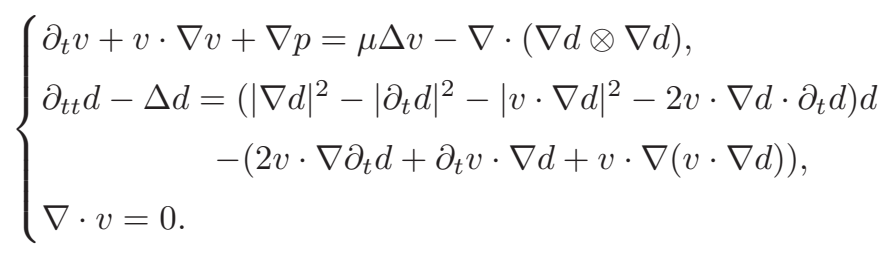

We have seven nonlinear terms in the equations for the orientation field, which make the whole argument a little annoying.

Now let us take a look at the invariant groups of the system (2.1). Suppose that $(u(t, x), d(t, x))$ is a solution of (2.1), then one can check that $\left(Q^{\top} u(t, Q x), d(t, Q x)\right)$ is also a solution of (2.1) for any 
orthogonal matrix $Q$. We choose $Q$ to be one parameter group generated by anti-symmetric matrices:

$$
Q=e^{\lambda A_{i}}, \quad \forall 1 \leq i \leq 3
$$

where

$$
\begin{aligned}
& A_{1}=e_{2} \otimes e_{3}-e_{3} \otimes e_{2}, \\
& A_{2}=e_{3} \otimes e_{1}-e_{1} \otimes e_{3}, \\
& A_{3}=e_{1} \otimes e_{2}-e_{2} \otimes e_{1} .
\end{aligned}
$$

The perturbed angular momentum operators are defined as infinitesimal generators of the orthogonal groups:

$$
\left\{\begin{array}{l}
\widetilde{\Omega}_{i} v=\Omega_{i} v+A_{i} v \\
\widetilde{\Omega}_{i} d=\Omega_{i} d
\end{array}\right.
$$

where $\Omega=\left(\Omega_{1}, \Omega_{2}, \Omega_{3}\right)$ is the rotational gradient operator defined by

$$
\Omega=x \wedge \nabla
$$

Schematically, we have the commutation:

$$
[\partial, \Omega]=\partial
$$

where $\partial$ means the span of $\left\{\partial_{t}, \partial_{1}, \partial_{2}, \partial_{3}\right\}$. (2.2) and the following commutation (2.4) will be often used implicitly.

Putting $\left(Q^{\top} u(t, Q x), d(t, Q x)\right)$ into (2.1), differentiating them with respect to $\lambda$ and taking $\lambda \rightarrow 0$, we have

$$
\left\{\begin{aligned}
\partial_{t} \widetilde{\Omega} v+\widetilde{\Omega} v & \nabla v+v \cdot \nabla \widetilde{\Omega} v+\nabla \widetilde{\Omega} p \\
= & \mu \Delta \widetilde{\Omega} v-\nabla \cdot(\nabla \widetilde{\Omega} d \otimes \nabla d)-\nabla \cdot(\nabla d \otimes \nabla \widetilde{\Omega} d), \\
\partial_{t t} \widetilde{\Omega} d-\Delta \widetilde{\Omega} d=2\left(\nabla \widetilde{\Omega} d \cdot \nabla d-\partial_{t} \widetilde{\Omega} d \cdot \partial_{t} d\right) d & \\
- & \left(2 \widetilde{\Omega} v \cdot \nabla d \cdot \partial_{t} d+2 v \cdot \nabla \widetilde{\Omega} d \cdot \partial_{t} d+2 v \cdot \nabla d \cdot \partial_{t} \widetilde{\Omega} d\right) d \\
& -2[(\widetilde{\Omega} v \cdot \nabla) d \cdot(v \cdot \nabla) d+(v \cdot \nabla) \widetilde{\Omega} d \cdot(v \cdot \nabla) d] d \\
& +\left(|\nabla d|^{2}-\left|\partial_{t} d\right|^{2}-|v \cdot \nabla d|^{2}-2 v \cdot \nabla d \cdot \partial_{t} d\right) \widetilde{\Omega} d \\
& -\left(2 \widetilde{\Omega} v \cdot \nabla \partial_{t} d+2 v \cdot \nabla \partial_{t} \widetilde{\Omega} d+\partial_{t} \widetilde{\Omega} v \cdot \nabla d+\partial_{t} v \cdot \nabla \widetilde{\Omega} d\right) \\
& -[\widetilde{\Omega} v \cdot \nabla(v \cdot \nabla d)+v \cdot \nabla(\widetilde{\Omega} v \cdot \nabla d)+v \cdot \nabla(v \cdot \nabla \widetilde{\Omega} d)] . \\
\nabla \cdot \widetilde{\Omega} v=0 . &
\end{aligned}\right.
$$

Next, we try to apply the scaling operator

$$
S=t \partial_{t}+r \partial_{r}
$$

onto the system. Unfortunately, the system (2.1) does not have any scaling invariance (nor Lorentz invariance). Inspired by 3, 11, however, we are still able to use the scaling operator under this circumstance.

Applying $S+1$ onto (2.1 $1,2.1)_{3}$, and applying $S+2$ onto $(2.1)_{2}$, thanks to the commutation:

$$
(S+1) \partial=\partial S, \quad(S+2) \partial^{2}=\partial^{2} S,
$$


we can derive by direct calculations that

$$
\left\{\begin{aligned}
\partial_{t} S v+S v & \nabla v+v \cdot \nabla S v+\nabla S p \\
= & \mu \Delta(S-1) v-\nabla \cdot(\nabla(S-1) d \otimes \nabla d)-\nabla \cdot(\nabla d \otimes \nabla(S-1) d), \\
\partial_{t t} S d-\Delta S d=2\left(\nabla S d \cdot \nabla d-\partial_{t} S d \cdot \partial_{t} d\right) d & \\
- & \left(2 S v \cdot \nabla d \cdot \partial_{t} d+2 v \cdot \nabla S d \cdot \partial_{t} d+2 v \cdot \nabla d \cdot \partial_{t} S d\right) d \\
- & 2[(S v \cdot \nabla) d \cdot(v \cdot \nabla) d+(v \cdot \nabla) S d \cdot(v \cdot \nabla) d] d \\
& +\left(|\nabla d|^{2}-\left|\partial_{t} d\right|^{2}-|v \cdot \nabla d|^{2}-2 v \cdot \nabla d \cdot \partial_{t} d\right) S d \\
- & \left(2 S v \cdot \nabla \partial_{t} d+2 v \cdot \nabla \partial_{t} S d+\partial_{t} S v \cdot \nabla d+\partial_{t} v \cdot \nabla S d\right) \\
- & {[S v \cdot \nabla(v \cdot \nabla d)+v \cdot \nabla(S v \cdot \nabla d)+v \cdot \nabla(v \cdot \nabla S d)] . }
\end{aligned}\right.
$$

Note that there are some commutators in the equation for velocity in (2.5) due to the appearance of viscosity. This is different from the application of rotation operator.

Now we are going to apply compositions of generalized operators. Let

$$
\Gamma \in\left\{\partial_{t}, \partial_{1}, \partial_{2}, \partial_{3}, \widetilde{\Omega}_{1}, \widetilde{\Omega}_{2}, \widetilde{\Omega}_{3}\right\}
$$

and $Z^{a}=S^{a_{1}} \Gamma^{a^{\prime}}$, where $a=\left(a_{1}, a_{2}, \ldots, a_{8}\right)=\left(a_{1}, a^{\prime}\right), \Gamma^{a^{\prime}}=\Gamma^{a_{2}} \Gamma^{a_{3}} \ldots \Gamma^{a_{8}}$. Using the reduction argument, we can use (2.3) and (2.5) to derive that

$$
\left\{\begin{array}{l}
\partial_{t} Z^{a} v-\mu \Delta(S-1)^{a_{1}} \Gamma^{a^{\prime}} v=f_{a}^{1} \\
\partial_{t t} Z^{a} d-\Delta Z^{a} d=f_{a}^{2} \\
\nabla \cdot Z^{a} v=0
\end{array}\right.
$$

where

$$
\left\{\begin{aligned}
f_{a}^{1}= & -\sum_{b+c=a} C_{a}^{b} Z^{b} v \cdot \nabla Z^{c} v-\nabla Z^{a} p \\
& -\sum_{b+c=a} C_{a}^{b} \nabla \cdot\left(\nabla(S-1)^{b_{1}} \Gamma^{b^{\prime}} d \otimes \nabla(S-1)^{c_{1}} \Gamma^{c^{\prime}} d\right), \\
f_{a}^{2}= & \sum_{b+c+e=a} C_{a}^{b, c}\left(\nabla Z^{b} d \cdot \nabla Z^{c} d-\partial_{t} Z^{b} d \cdot \partial_{t} Z^{c} d\right) Z^{e} d \\
& -\sum_{b+c+e+f=a} C_{a}^{b, c, e}\left[2\left(Z^{b} v \cdot \nabla\right) Z^{c} d \cdot \partial_{t} Z^{e} d\right] Z^{f} d \\
& -\sum_{b+c+e+f+g=a} C_{a}^{b, c, e, f}\left[\left(Z^{b} v \cdot \nabla\right) Z^{c} d \cdot\left(\left(Z^{e} v \cdot \nabla\right) Z^{f} d\right)\right] Z^{g} d \\
& -\sum_{b+c+e=a} C_{a}^{b, c} Z^{b} v \cdot \nabla\left(Z^{c} v \cdot \nabla Z^{e} d\right) \\
& -\sum_{b+c=a} C_{a}^{b}\left(2 Z^{b} v \cdot \nabla \partial_{t} Z^{c} d+\partial_{t} Z^{b} v \cdot \nabla Z^{c} d\right) .
\end{aligned}\right.
$$

Here $C_{a}^{b}, C_{a}^{b, c}, C_{a}^{b, c, e}$ and $C_{a}^{b, c, e, f}$ are multinomial coefficients:

$$
\begin{aligned}
& C_{a}^{b}=\frac{a !}{b !(a-b) !}, \quad C_{a}^{b, c}=\frac{a !}{b ! c !(a-b-c) !} \\
& C_{a}^{b, c, e}=\frac{a !}{b ! c ! e !(a-b-c-e) !}, \quad C_{a}^{b, c, e, f}=\frac{a !}{b ! c ! e ! f !(a-b-c-e-f) !} .
\end{aligned}
$$

The above commutation relations (2.6)-(2.7) are the starting points of this paper.

\subsection{Some notations}

Throughout this paper, we use the generalized energy defined by

$$
E_{\kappa}^{v}(t)=\left\|Z^{\kappa} v(t, \cdot)\right\|_{L^{2}}^{2}, \quad E_{\kappa+1}^{d}(t)=\left\|\partial Z^{\kappa} d(t, \cdot)\right\|_{L^{2}}^{2} .
$$


where $Z^{\kappa} v=\left\{Z^{a} v:|a| \leq \kappa\right\}, Z^{\kappa} d=\left\{Z^{a} d:|a| \leq \kappa\right\}$.

We also use the weighted energy norm of Klainerman-Sideris [17]:

$$
X_{\kappa}^{d}(t)=\left\|\langle r-t\rangle \partial^{2} Z^{\kappa-2} d\right\|_{L^{2}}^{2},
$$

in which $\langle\sigma\rangle=\sqrt{1+\sigma^{2}}$, for $\kappa \geq 2$.

To describe the space of initial data, we introduce (see [41])

$$
\Lambda=\left\{\nabla, \widetilde{\Omega}, r \partial_{r}\right\}
$$

and

$$
H_{\Lambda}^{\kappa}=\left\{(f, g, h): \sum_{|a| \leq \kappa}\left\|\Lambda^{a} f\right\|_{L^{2}}+\left\|\nabla \Lambda^{a} g\right\|_{L^{2}}+\left\|\Lambda^{a} h\right\|_{L^{2}}<\infty\right\},
$$

with the norm

$$
\|(f, g, h)\|_{H_{\Lambda}^{\kappa}}=\sum_{|a| \leq \kappa}\left(\left\|\Lambda^{a} f\right\|_{L^{2}}+\left\|\nabla \Lambda^{a} g\right\|_{L^{2}}+\left\|\Lambda^{a} h\right\|_{L^{2}}\right),
$$

for the vector of $f, g$ and $h$.

Throughout the whole paper, we will use $A \lesssim B$ to denote $A \leq C B$ for some positive absolute constant $C$, whose meaning may change from line to line. We remark that, without specification, the constant depends only on $\kappa$, but not on $t$.

\subsection{Ansatz for the method of continuity}

The local well-posedness has been proved recently in 10. To extend the local solution to be a global one, we need to show the uniform estimate in time.

We make the following ansatz for the generalized energy:

$$
E_{\kappa}^{v} \leq C \epsilon, \quad E_{\kappa+1}^{d} \leq C \epsilon\langle t\rangle^{\delta}, \quad E_{\kappa-1}^{d} \leq C \epsilon,
$$

for $\kappa \geq 9$, which is (1.2) in Theorem 1.1. Under the above a priori assumption, we first show that the weighted $L^{2}$ norm $X_{\kappa}$ can be controlled by the generalized energy:

$$
X_{\kappa-1}^{d} \lesssim E_{\kappa-1}^{d}, \quad X_{\kappa+1}^{d} \lesssim E_{\kappa+1}^{d},
$$

see Section 3. Next, we show the decay estimate for the velocity in Section 4:

$$
\begin{aligned}
& \left\|\langle t\rangle^{\frac{3}{4}} v\right\|_{L_{x}^{\infty}} \lesssim \epsilon^{\frac{1}{2}}, \quad\left\|\langle t\rangle^{\frac{5}{4}} \nabla v\right\|_{L_{x}^{\infty}} \lesssim \epsilon^{\frac{1}{2}}, \\
& \left\|\langle t\rangle^{\frac{3}{2}} \nabla^{a} v\right\|_{L_{x}^{\infty}} \leq \epsilon^{\frac{1}{2}}(\ln \langle t\rangle)^{\frac{1}{2}}, \quad \forall 2 \leq|a| \leq \kappa-3, \\
& \left\|\partial_{t} v\right\|_{L_{x}^{\infty}} \lesssim\langle t\rangle^{-\frac{3}{2}}(\ln \langle t\rangle)^{\frac{1}{2}} \epsilon^{\frac{1}{2}} .
\end{aligned}
$$

Then, we can finally close the energy estimates by establishing the following inequalities:

$$
\begin{aligned}
E_{\kappa}^{v}(t)+ & \mu\left\|\nabla Z^{k} v(\cdot, \cdot)\right\|_{L_{t}^{2} L_{x}^{2}}^{2} \\
\lesssim & E_{\kappa}^{v}(0)+\int_{0}^{t}\langle\tau\rangle^{-2} E_{\kappa+1}^{d}(\tau) E_{\kappa-1}^{d}(\tau) \mathrm{d} \tau+\left\|\nabla Z^{k} v(\cdot, \cdot)\right\|_{L_{t}^{2} L_{x}^{2}}^{2} E_{\kappa}^{v}(t) . \\
\frac{d}{d t} E_{\kappa+1}^{d} \lesssim\langle t\rangle^{-1}\left(E_{\kappa}^{v}+E_{\kappa-1}^{d}\right)^{\frac{1}{2}} E_{\kappa+1}^{d}+\left\|\nabla Z^{\kappa} v\right\|_{L^{2}}^{2} E_{\kappa+1}^{d} & \\
& +\langle t\rangle^{-1}\left\|\nabla Z^{\kappa} v\right\|_{L^{2}} E_{\kappa+1}^{d}+\left\|\partial_{t} v\right\|_{L^{\infty}} E_{\kappa+1}^{d} . \\
\frac{d}{d t} E_{\kappa-1}^{d} \lesssim\langle t\rangle^{-1}\left\|\nabla Z^{\kappa} v\right\|_{L^{2}}^{\frac{1}{2}}\left(E_{\kappa}^{v}\right)^{\frac{1}{4}}\left(E_{\kappa-1}^{d} E_{\kappa+1}^{d}\right)^{\frac{1}{2}} & \\
& +\langle t\rangle^{-\frac{3}{2}} E_{\kappa-1}^{d}\left(E_{\kappa+1}^{d}\right)^{\frac{1}{2}}+\left\|\nabla Z^{\kappa} v\right\|_{L^{2}}^{2} E_{\kappa-1}^{d} .
\end{aligned}
$$

This is the main topic in Section 5.

With the help of above estimates (2.8)-(2.11), we can prove the main theorem of the paper. 
Proof of Theorem 1.1. It suffices to show that under the bootstrap assumption:

$$
E_{\kappa+1}^{d}(t) \leq C_{0} \epsilon\langle t\rangle^{\delta}, \quad E_{\kappa}^{v}(t)+\frac{1}{2} \mu\left\|\nabla Z^{k} v(\cdot, \cdot)\right\|_{L_{t}^{2} L_{x}^{2}}^{2}+E_{\kappa-1}^{d}(t) \leq C_{0} \epsilon \ll 1,
$$

for some positive $C_{0}>0,0<\delta<\frac{1}{2}$ depending on $\kappa$ and $\epsilon_{0}$ uniformly for $0 \leq t<T$, we can derive a stronger estimate:

$$
E_{\kappa+1}^{d}(t) \leq \frac{1}{2} C_{0} \epsilon\langle t\rangle^{\delta}, \quad E_{\kappa}^{v}(t)+\frac{1}{2} \mu\left\|\nabla Z^{k} v(\cdot, \cdot)\right\|_{L_{t}^{2} L_{x}^{2}}^{2}+E_{\kappa-1}^{d}(t) \leq \frac{1}{2} C_{0} \epsilon .
$$

Then we can use continuity argument to extend life span of the solutions.

Firstly, under the assumption of (2.12), we have from (2.9) that

$$
\begin{aligned}
E_{\kappa}^{v}(t)+\frac{1}{2} \mu\left\|\nabla Z^{k} v(\cdot, \cdot)\right\|_{L_{t}^{2} L_{x}^{2}}^{2} & \leq C E_{\kappa}^{v}(0)+C \int_{0}^{t}\langle\tau\rangle^{-2} E_{\kappa+1}^{d}(\tau) E_{\kappa-1}^{d}(\tau) \mathrm{d} \tau \\
& \leq C E_{\kappa}^{v}(0)+C \int_{0}^{t}\langle\tau\rangle^{\delta-2} C_{0}^{2} \epsilon^{2} \mathrm{~d} \tau \\
& \leq C \epsilon+C C_{0}^{2} \epsilon^{2} .
\end{aligned}
$$

Let $C_{0}$ and $\epsilon$ such that

$$
\max \{8,8 C\} \leq C_{0}, \quad 8 C C_{0} \epsilon \leq 1,
$$

then $E_{\kappa}^{v}(t)+\frac{1}{2} \mu\left\|\nabla Z^{k} v(\cdot, \cdot)\right\|_{L_{t}^{2} L_{x}^{2}}^{2} \leq \frac{1}{4} C_{0} \epsilon$.

Secondly, under the assumption of (2.12), we can derive from (2.10) that

$$
\frac{d}{d t} E_{\kappa+1}^{d} \leq C\left(\langle t\rangle^{-1}\left(E_{\kappa}^{v}+E_{\kappa-1}^{d}\right)^{\frac{1}{2}}+\left\|\nabla Z^{\kappa} v\right\|_{L^{2}}^{2}+\langle t\rangle^{-1}\left\|\nabla Z^{\kappa} v\right\|_{L^{2}}+\left\|\partial_{t} v\right\|_{L^{\infty}}\right) E_{\kappa+1}^{d} .
$$

Then, Gronwall inequality gives us that

$$
\begin{aligned}
E_{\kappa+1}^{d}(t) \leq & E_{\kappa+1}^{d}(0) \exp \left(C \int_{0}^{t}\langle\tau\rangle^{-1}\left(E_{\kappa}^{v}+E_{\kappa-1}^{d}\right)^{\frac{1}{2}}(\tau)+\left\|\nabla Z^{\kappa} v(\tau)\right\|_{L^{2}}^{2}\right. \\
& \left.+\langle\tau\rangle^{-1}\left\|\nabla Z^{\kappa} v(\tau)\right\|_{L^{2}}+\left\|\partial_{t} v\right\|_{L^{\infty}} d \tau\right) \\
\leq & \epsilon \exp \left(C\left(C_{0} \epsilon\right)^{\frac{1}{2}} \ln \langle t\rangle+C C_{0} \epsilon+C\left(C_{0} \epsilon\right)^{\frac{1}{2}}+C \epsilon^{\frac{1}{2}}\right) \\
\leq & \epsilon \exp \left(C\left(C_{0} \epsilon\right)^{\frac{1}{2}}\right)\langle t\rangle^{C C_{0}^{\frac{1}{2}} \epsilon^{\frac{1}{2}}} .
\end{aligned}
$$

Taking $\epsilon$ small enough such that

$$
\exp \left(C\left(C_{0} \epsilon\right)^{\frac{1}{2}}\right) \leq \frac{1}{2} C_{0}, \quad C C_{0}^{\frac{1}{2}} \epsilon^{\frac{1}{2}} \leq \delta \leq \frac{1}{2},
$$

we have $E_{\kappa+1}^{d}(t) \leq \frac{1}{2} C_{0} \epsilon\langle t\rangle^{\delta}$.

Finally, using Holder's inequality, we derive from (2.11) that

$$
\begin{aligned}
\frac{d}{d t} E_{\kappa-1}^{d} \leq & C\left(\langle t\rangle^{-1}\left\|\nabla Z^{\kappa} v\right\|_{L^{2}}^{\frac{1}{2}}\left(E_{\kappa+1}^{d}\right)^{\frac{1}{2}}+\langle t\rangle^{-\frac{3}{2}}\left(E_{\kappa+1}^{d}\right)^{\frac{1}{2}}+\left\|\nabla Z^{\kappa} v\right\|_{L^{2}}^{2}\right) E_{\kappa-1}^{d} \\
& +C\langle t\rangle^{-1}\left\|\nabla Z^{\kappa} v\right\|_{L^{2}}^{\frac{1}{2}}\left(E_{\kappa}^{v}\right)^{\frac{1}{2}}\left(E_{\kappa+1}^{d}\right)^{\frac{1}{2}}
\end{aligned}
$$

Then the Gronwall inequality gives us that

$$
E_{\kappa-1}^{d}(t) \leq\left(E_{\kappa-1}^{d}(0)+C \int_{0}^{t}\langle\tau\rangle^{-1}\left\|\nabla Z^{\kappa} v(\tau)\right\|_{L^{2}}^{\frac{1}{2}}\left(E_{\kappa}^{v}(\tau)\right)^{\frac{1}{2}}\left(E_{\kappa+1}^{d}(\tau)\right)^{\frac{1}{2}} d \tau\right)
$$




$$
\begin{aligned}
& \cdot \exp \left(C \int_{0}^{t}\langle\tau\rangle^{-1}\left\|\nabla Z^{\kappa} v(\tau)\right\|_{L^{2}}^{\frac{1}{2}}\left(E_{\kappa+1}^{d}(\tau)\right)^{\frac{1}{2}}+\langle\tau\rangle^{-\frac{3}{2}}\left(E_{\kappa+1}^{d}(\tau)\right)^{\frac{1}{2}}+\left\|\nabla Z^{\kappa} v(\tau)\right\|_{L^{2}}^{2} d \tau\right) \\
& \leq\left(\epsilon+C\left(C_{0} \epsilon\right)^{\frac{3}{2}}\right) \exp \left(C C_{0} \epsilon+C C_{0}^{\frac{1}{2}} \epsilon^{\frac{1}{2}}\right) .
\end{aligned}
$$

As $c_{0}>8$, we can choose $\epsilon$ small enough such that

$$
\left(\epsilon+C\left(C_{0} \epsilon\right)^{\frac{3}{2}}\right) \exp \left(C C_{0} \epsilon+C C_{0}^{\frac{1}{2}} \epsilon^{\frac{1}{2}}\right) \leq \frac{1}{4} C_{0} \epsilon,
$$

then we have $E_{\kappa-1}^{d}(t) \leq \frac{1}{4} C_{0} \epsilon$.

Therefore, if we choose appropriate $C_{0}$ and small $\epsilon$ such that (2.14), (2.15) and (2.16) holds, then a better estimates (2.13) can be obtained. Thus the theorem is proved.

\subsection{Preliminary Weighted Estimates}

In this section, we list a few weighted estimates, which will be frequently used through out this paper.

First, we give two weighted $L^{\infty}-L^{2}$ estimates of the unknown near the light cone. They are essentially due to Klainerman and Sideris [17.

Lemma 2.1. Let $u \in H^{2}\left(\mathbb{R}^{3}\right)$, then there hold

$$
\begin{aligned}
\langle r\rangle^{1 / 2}|u(x)| & \lesssim \sum_{|\alpha| \leq 1}\left\|\nabla \widetilde{\Omega}^{\alpha} u\right\|_{L^{2}}, \\
\langle r\rangle|u(x)| & \lesssim \sum_{|\alpha| \leq 1}\left\|\partial_{r} \widetilde{\Omega}^{\alpha} u\right\|_{L^{2}}^{1 / 2} \cdot \sum_{|\alpha| \leq 2}\left\|\widetilde{\Omega}^{\alpha} u\right\|_{L^{2}}^{1 / 2},
\end{aligned}
$$

provided the right hand side is finite.

Proof. For (2.17), see Lemma 4.2 in [17. For (2.18), see Lemma 3.3 in [41].

Next, we present some weighted $L^{\infty}-L^{2}$ estimate away from the light cone.

Lemma 2.2. Let $u \in H^{2}\left(\mathbb{R}^{3}\right)$, then there hold

$$
\begin{aligned}
& \langle t\rangle\|u(t, \cdot)\|_{L^{\infty}(r \leq\langle t\rangle / 2)} \lesssim\|u\|_{L^{2}}+\|\langle t-r\rangle \nabla u\|_{L^{2}}+\left\|\langle t-r\rangle \nabla^{2} u\right\|_{L^{2}}, \\
& \langle t\rangle\|u(t, \cdot)\|_{L^{6}(r \leq\langle t\rangle / 2)} \lesssim\|u\|_{L^{2}}+\|\langle t-r\rangle \nabla u\|_{L^{2}}, \\
& \langle t\rangle^{\frac{1}{2}}\|u(t, \cdot)\|_{L^{3}(r \leq\langle t\rangle / 2)} \lesssim\|u\|_{L^{2}\left(\mathbb{R}^{3}\right)}^{\frac{1}{2}}\left(\|\langle r-t\rangle \nabla u\|_{L^{2}\left(\mathbb{R}^{3}\right)}+\|u\|_{L^{2}\left(\mathbb{R}^{3}\right)}\right)^{\frac{1}{2}},
\end{aligned}
$$

provided the right hand side is finite.

The first inequality (2.19) comes from [26] of Lemma 4.3.

Remark 2.1. This lemma depends on the fact that the spatial dimension is three or higher. In the two dimensional case, the conclusion would be weaker.

Proof. These three inequalities follow from the following Sobolev imbedding respectively: $\|u\|_{L^{\infty}\left(\mathbb{R}^{3}\right)} \lesssim$ $\|\nabla u\|_{L^{2}\left(\mathbb{R}^{3}\right)}^{\frac{1}{2}}\left\|\nabla^{2} u\right\|_{L^{2}\left(\mathbb{R}^{3}\right)}^{\frac{1}{2}},\|u\|_{L^{6}\left(\mathbb{R}^{3}\right)} \lesssim\|\nabla u\|_{L^{2}\left(\mathbb{R}^{3}\right)}$, and $\|u\|_{L^{3}\left(\mathbb{R}^{3}\right)} \lesssim\|u\|_{L^{2}\left(\mathbb{R}^{3}\right)}^{\frac{1}{2}}\|\nabla u\|_{L^{2}\left(\mathbb{R}^{3}\right)}^{\frac{1}{2}}$, providing $u \in L^{2}\left(\mathbb{R}^{3}\right)$. Due to the similarity of these inequalities, we only present a detailed proof for the third one.

Choose a radial cut-off function $\phi \in C^{\infty}\left(\mathbb{R}^{3}\right)$ which satisfies

$$
\phi(x)=\left\{\begin{array}{ll}
1, & \text { if } r \leq \frac{1}{2} \\
0, & \text { if } r \geq \frac{2}{3}
\end{array} \quad, \quad|\nabla \phi| \lesssim 1 .\right.
$$


For each fixed $t \geq 1$, let $\phi^{t}(x)=\phi(x /\langle t\rangle)$. Clearly, one has

$$
\phi^{t}(x) \equiv 1 \text { for } r \leq \frac{\langle t\rangle}{2}, \quad \phi^{t}(x) \equiv 0 \text { for } r \geq \frac{2\langle t\rangle}{3}
$$

and

$$
\left|\nabla \phi^{t}(x)\right| \lesssim\langle t\rangle^{-1}
$$

Consequently,

$$
\begin{aligned}
\|u\|_{L^{3}(r \leq\langle t\rangle / 2)} & \leq\left\|\phi^{t} u\right\|_{L^{3}\left(\mathbb{R}^{3}\right)} \\
& \lesssim\left\|\phi^{t} u\right\|_{L^{2}\left(\mathbb{R}^{3}\right)}^{\frac{1}{2}}\left(\left\|\phi^{t} \nabla u\right\|_{L^{2}\left(\mathbb{R}^{3}\right)}+\langle t\rangle^{-1}\|u\|_{L^{2}\left(\mathbb{R}^{3}\right)}\right)^{\frac{1}{2}} \\
& \lesssim\langle t\rangle^{-\frac{1}{2}}\|u\|_{L^{2}\left(\mathbb{R}^{3}\right)}^{\frac{1}{2}}\left(\|\langle r-t\rangle \nabla u\|_{L^{2}\left(\mathbb{R}^{3}\right)}+\|u\|_{L^{2}\left(\mathbb{R}^{3}\right)}\right)^{\frac{1}{2}}
\end{aligned}
$$

which yields (2.21).

Now we state two lemmas of weighted estimates using the structure of wave type equations, which can be found in [17] and [24]. We only state them without giving details of the proof.

Lemma 2.3. There holds

$$
X_{2}^{d} \lesssim E_{2}^{d}+\left\|\langle t+r\rangle\left(\partial_{t}^{2}-\Delta\right) d\right\|_{L^{2}}
$$

provided the right hand side is finite.

Proof. See Lemma 2.3 and Lemma 3.1 in [17.

Near the light cone, the good unknown $\left(\partial_{t}+\partial_{r}\right) d$ has better decay. The following lemma comes from 24 where the two dimension case was proved. Indeed, it holds for all dimension $n \geq 2$.

Lemma 2.4. For $\frac{\langle t\rangle}{2} \leq r$, there holds

$$
\left.\langle t\rangle\left|\left(\partial_{t}+\partial_{r}\right) \partial d\right| \lesssim|\nabla d|+|\nabla Z d|+t \mid\left(\partial_{t}^{2}-\Delta\right) d\right) \mid .
$$

Proof. See Lemma 3.4 in [24]. The proof given in 24] is for $\frac{\langle t\rangle}{2} \leq r \leq \frac{5\langle t\rangle}{2}$ with space dimension $n=2$. However, one can easily check that the proof is valid for $r \geq \frac{\langle t\rangle}{2}$ and $n \geq 2$.

\section{Estimates of the weighted $L^{2}$ norm}

This section is devoted to the estimate of weighted $L^{2}$ norm $X_{\kappa}$. To this end, we need to estimate the $L^{2}$ norm of $f_{a}^{2}$ with some weights.

Lemma 3.1. For all multi-index a, there holds

$$
\begin{aligned}
\| & (t+r) f_{a}^{2} \|_{L^{2}}^{2} \\
\lesssim & E_{|a|+1}^{d}\left(X_{[|a| / 2]+3}^{d}+E_{[|a| / 2]+3}^{d}\right)\left(E_{[|a| / 2]+3}^{d}+1\right) \\
& +E_{|a|+1}^{v}\left(E_{[|a| / 2]+3}^{v}+1\right)\left(E_{[|a| / 2]+4}^{d}+X_{[|a| / 2]+4}^{d}\right) \\
& +\left(E_{|a|+2}^{d}+X_{|a|+2}^{d}\right) E_{[|a| / 2]+3}^{v}\left(E_{[|a| / 2]+3}^{v}+1\right) \\
& +\left(E_{|a|}^{v} E_{[|a| / 2]+3}^{d}+E_{[|a| / 2]+2}^{v} E_{|a|+1}^{d}\right)\left(E_{[|a| / 2]+3}^{v}+1\right)\left(E_{[|a| / 2]+3}^{d}+X_{[|a| / 2]+3}^{d}\right)\left(E_{[|a| / 2]+3}^{d}+1\right),
\end{aligned}
$$

provided the right hand side is finite. 
Proof. Recalling the definition of $f_{a}^{2}$ in (2.7), we write

$$
\begin{aligned}
& \sum_{b+c+e=a} C_{a}^{b, c}\left\|(t+r)\left(\nabla Z^{b} d \cdot \nabla Z^{c} d-\partial_{t} Z^{b} d \cdot \partial_{t} Z^{c} d\right) Z^{e} d\right\|_{L^{2}}^{2} \\
& +\sum_{b+c=a} C_{a}^{b}\left\|(t+r)\left(2 Z^{b} v \cdot \nabla \partial_{t} Z^{c} d\right)\right\|_{L^{2}}^{2} \\
& +\sum_{b+c=a} C_{a}^{b}\left\|(t+r)\left(\partial_{t} Z^{b} v \cdot \nabla Z^{c} d\right)\right\|_{L^{2}}^{2} \\
& +\sum_{b+c+e=a} C_{a}^{b, c}\left\|(t+r) Z^{b} v \cdot \nabla\left(Z^{c} v \cdot \nabla Z^{e} d\right)\right\|_{L^{2}}^{2} \\
& \left.+\sum_{b+c+e+f+g=a} C_{a}^{b, c, e, f} \|(t+r)\left(Z^{b} v \cdot \nabla\right) Z^{c} d \cdot\left(\left(Z^{e} v \cdot \nabla\right) Z^{f} d\right)\right] Z^{g} d \|_{L^{2}}^{2} \\
& +\sum_{b+c+e+f=a} C_{a}^{b, c, e}\left\|(t+r)\left[2\left(Z^{b} v \cdot \nabla\right) Z^{c} d \cdot \partial_{t} Z^{e} d\right] Z^{f} d\right\|_{L^{2}}^{2} \\
& =H_{1}+H_{2}+H_{3}+H_{4}+H_{5}+H_{6} .
\end{aligned}
$$

In the sequel, we will focus our mind on $H_{1}, H_{2}$ and $H_{3}$, since these terms contain quadratic terms. The remain terms $H_{4}, H_{5}, H_{6}$ are all cubic or higher order ones whose estimates are similar and easier.

We first estimate $H_{1}$ :

$$
\begin{aligned}
H_{1} \lesssim\left\|(t+r)\left|\partial Z^{|a|} d\left\|\partial Z^{[|a| / 2]} d\right\| Z^{[|a| / 2]} d\right|\right\|_{L^{2}}^{2} & \\
& +\sum_{[a / 2] \leq|b| \leq|a|}\left\|(t+r)\left|\partial Z^{[|a| / 2]} d\right|^{2}\left|Z^{b} d\right|\right\|_{L^{2}}^{2}
\end{aligned}
$$

If $r \geq\langle t\rangle / 2$, by (2.18), the right hand side of (3.1) can be controlled by

$$
\begin{aligned}
& \left\|\partial Z^{|a|} d\right\|_{L^{2}}^{2}\left\|r \partial Z^{[|a| / 2]} d\right\|_{L^{\infty}(r \geq\langle t\rangle / 2)}^{2}\left\|Z^{[|a| / 2]} d\right\|_{L^{\infty}}^{2} \\
& +\sum_{[a / 2] \leq|b| \leq|a|}\left\|r \partial Z^{[a / 2]} d\right\|_{L^{\infty}(r \geq\langle t\rangle / 2)}^{2}\left\|\partial Z^{[a / 2]} d\right\|_{L^{3}(r \geq\langle t\rangle / 2)}^{2}\left\|Z^{b} d\right\|_{L^{6}}^{2} \\
& \lesssim E_{|a|+1}^{d} E_{[|a| / 2]+3}^{d}\left(1+E_{[|a| / 2]+3}^{d}\right) .
\end{aligned}
$$

Otherwise, if $r \leq\langle t\rangle / 2$, by (2.19) and (2.20), the right hand side of (3.1) can be controlled by

$$
\begin{aligned}
& \left\|\partial Z^{|a|} d\right\|_{L^{2}}^{2}\left\|\langle t\rangle \partial Z^{[|a| / 2]} d\right\|_{L^{\infty}(r \leq\langle t\rangle / 2)}\left\|Z^{[|a| / 2]} d\right\|_{L^{\infty}}^{2} \\
& +\sum_{[a / 2] \leq|b| \leq|a|}\left\|\partial Z^{[a / 2]} d\right\|_{L^{6}}^{2}\left\|\langle t\rangle \partial Z^{[a / 2]} d\right\|_{L^{6}(r \leq\langle t\rangle / 2)}^{2}\left\|Z^{b} d\right\|_{L^{6}}^{2} \\
& \lesssim E_{|a|+1}^{d}\left(X_{[|a| / 2]+3}^{d}+E_{[|a| / 2]+2}^{d}\right)\left(E_{[|a| / 2]+2}^{d}+1\right) .
\end{aligned}
$$

Thus we conclude that

$$
H_{1} \lesssim E_{|a|+1}^{d}\left(X_{[|a| / 2]+3}^{d}+E_{[|a| / 2]+3}^{d}\right)\left(E_{[|a| / 2]+3}^{d}+1\right)
$$

For $H_{2}$, we have:

$$
H_{2}=4 \sum_{b+c=a} C_{a}^{b}\left\|(t+r) Z^{b} v \cdot \nabla \partial_{t} Z^{c} d\right\|_{L^{2}}^{2} .
$$

When $r \geq\langle t\rangle / 2$, by (2.18), we can estimate the right hand side of (3.2) as

$$
\left\|r Z^{[|a| / 2]} v\right\|_{L^{\infty}(r \geq\langle t\rangle / 2)}^{2}\left\|\nabla \partial_{t} Z^{|a|} d\right\|_{L^{2}}^{2}+\left\|Z^{|a|} v\right\|_{L^{2}}^{2}\left\|r \nabla \partial_{t} Z^{[|a| / 2]} d\right\|_{L^{\infty}(r \geq\langle t\rangle / 2)}^{2}
$$




$$
\lesssim E_{[|a| / 2]+2}^{v} E_{|a|+2}^{d}+E_{|a|}^{v} E_{[|a| / 2]+4}^{d} .
$$

When $r \leq\langle t\rangle / 2$, using (2.19), the right hand side of (3.2) can be controlled by

$$
\begin{aligned}
& \left\|Z^{[|a| / 2]} v\right\|_{L^{\infty}}^{2}\left\|\langle t-r\rangle \nabla \partial_{t} Z^{|a|} d\right\|_{L^{2}}^{2}+\left\|Z^{|a|} v\right\|_{L^{2}}^{2}\left\|\langle t\rangle \nabla \partial_{t} Z^{|a|} d\right\|_{L^{\infty}(r \leq\langle t\rangle / 2)}^{2} \\
& \lesssim E_{[|a| / 2]+2}^{v} X_{|a|+2}^{d}+E_{|a|}^{v} X_{[|a| / 2]+4}^{d}+E_{|a|}^{v} E_{[|a| / 2]+2}^{d} .
\end{aligned}
$$

Hence we conclude that

$$
H_{2} \lesssim E_{[|a| / 2]+2}^{v} E_{|a|+2}^{d}+E_{|a|}^{v} E_{[|a| / 2]+4}^{d}+E_{[|a| / 2]+2}^{v} X_{|a|+2}^{d}+E_{|a|}^{v} X_{[|a| / 2]+4}^{d} .
$$

Then we consider

$$
H_{3} \lesssim\left\|( t + r ) \left|\partial_{t} Z^{|a|} v\left\|\left|\nabla Z^{[|a| / 2]} d\right|\right\|_{L^{2}}^{2}+\left\|\left|(t+r) \partial_{t} Z^{[|a| / 2]} v\left\|\left|\nabla Z^{|a|} d\right|\right\|_{L^{2}}^{2} .\right.\right.\right.\right.
$$

When $r \geq\langle t\rangle / 2$, similar to the estimate of (3.2), the right hand side of (3.3) can be bounded by

$$
E_{|a|+1}^{v} E_{[|a| / 2]+3}^{d}+E_{[|a| / 2]+3}^{v} E_{|a|+1}^{d} .
$$

For the case of $r \leq\langle t\rangle / 2$, with the help of (2.19) and (2.20), we can estimate the right hand side of (3.3) as

$$
\begin{aligned}
& \left\|\partial_{t} Z^{|a|} v\right\|_{L^{2}}^{2}\left\|\langle t\rangle \nabla Z^{[|a| / 2]} d\right\|_{L^{\infty}(r \leq\langle t\rangle / 2)}^{2}+\left\|\partial_{t} Z^{[|a| / 2]} v\right\|_{L^{3}}\left\|\langle t\rangle \nabla Z^{|a|} d\right\|_{L^{6}(r \leq\langle t\rangle / 2)}^{2} \\
& \lesssim E_{|a|+1}^{v}\left(X_{[|a| / 2]+3}^{d}+E_{[|a| / 2]+1}^{d}\right)+E_{[|a| / 2]+2}^{v}\left(X_{|a|+2}^{d}+E_{|a|+1}^{d}\right) .
\end{aligned}
$$

Therefore, we have

$$
H_{3} \lesssim E_{|a|+1}^{v}\left(X_{[|a| / 2]+3}^{d}+E_{[|a| / 2]+3}^{d}\right)+E_{[|a| / 2]+3}^{v}\left(X_{|a|+2}^{d}+E_{|a|+1}^{d}\right) .
$$

For $H_{4}, H_{5}$ and $H_{6}$, along the same line, we can obtain that

$$
\begin{aligned}
H_{4} & \lesssim E_{|a|+1}^{v} E_{[|a| / 2]+3}^{v}\left(E_{[|a| / 2]+4}^{d}+X_{[|a| / 2]+4}^{d}\right)+\left(X_{|a|+2}^{d}+E_{|a|+2}^{d}\right)\left(E_{[|a| / 2]+3}^{v}\right)^{2}, \\
H_{5} & \lesssim\left(E_{|a|}^{v} E_{[|a| / 2]+3}^{d}+E_{[|a| / 2]+2}^{v} E_{|a|+1}^{d}\right) E_{[|a| / 2]+3}^{v}\left(E_{[|a| / 2]+3}^{d}+X_{[|a| / 2]+3}^{d}\right)\left(E_{[|a| / 2]+3}^{d}+1\right), \\
H_{6} & \lesssim\left(E_{|a|}^{v} E_{[|a| / 2]+3}^{d}+E_{[|a| / 2]+2}^{v} E_{|a|+1}^{d}\right)\left(E_{[|a| / 2]+3}^{d}+X_{[|a| / 2]+3}^{d}\right)\left(E_{[|a| / 2]+3}^{d}+1\right) .
\end{aligned}
$$

Combining the estimates of $H_{1}, \ldots, H_{6}$ gives the lemma.

Now we show that $X_{\kappa}^{d}$ can be controlled by the generalized energy under certain small energy assumptions.

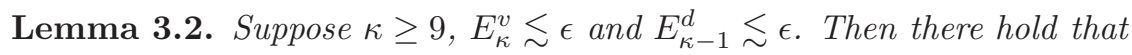

$$
X_{\kappa-1}^{d} \lesssim E_{\kappa-1}^{d}, \quad X_{\kappa+1}^{d} \lesssim E_{\kappa+1}^{d} .
$$

Proof. Let $\kappa \geq 9,|a|+2 \leq \kappa+1$, then one has $[a / 2]+4 \leq \kappa-1$. Before proving the lemma, we first show that under the assumption of $E_{\kappa}^{v} \lesssim \epsilon, E_{\kappa-1}^{d} \lesssim \epsilon$, there holds

$$
\begin{aligned}
X_{|a|+2}^{d} \lesssim E_{|a|+2}^{d} & +E_{|a|+1}^{d}\left(X_{[|a| / 2]+3}^{d}+E_{[|a| / 2]+3}^{d}\right) \\
& +E_{|a|+1}^{v}\left(E_{[|a| / 2]+4}^{d}+X_{[|a| / 2]+4}^{d}\right)+\left(E_{|a|+2}^{d}+X_{|a|+2}^{d}\right) E_{[|a| / 2]+3}^{v} .
\end{aligned}
$$

Actually, by Lemma 2.3, one has

$$
X_{|a|+2}^{d} \lesssim E_{|a|+2}^{d}+\sum_{|b| \leq|a|}\left\|(t+r) f_{b}^{2}\right\|_{L^{2}}^{2} .
$$


On the other hand, thanks to Lemma 3.1 and the assumption $E_{\kappa}^{v} \lesssim \epsilon, E_{\kappa-1}^{d} \lesssim \epsilon$, one can easily check that

$$
\begin{aligned}
\left\|(t+r) f_{a}^{2}\right\|_{L^{2}}^{2} \lesssim & E_{|a|+1}^{d}\left(X_{[|a| / 2]+3}^{d}+E_{[|a| / 2]+3}^{d}\right) \\
& \quad+E_{|a|+1}^{v}\left(E_{[|a| / 2]+4}^{d}+X_{[|a| / 2]+4}^{d}\right)+\left(E_{|a|+2}^{d}+X_{|a|+2}^{d}\right) E_{[|a| / 2]+3}^{v} .
\end{aligned}
$$

Thus (3.4) can be deduced directly.

Now we turn to the proof of the lemma. Let $|a|+2 \leq \kappa-1$, one can get by (3.4) that

$$
X_{\kappa-1}^{d} \lesssim E_{\kappa-1}^{d}+E_{\kappa-1}^{d}\left(E_{\kappa-1}^{d}+E_{\kappa-1}^{v}\right)+\left(E_{\kappa-1}^{d}+E_{\kappa-1}^{v}\right) X_{\kappa-1}^{d}
$$

Using the assumption of $E_{\kappa}^{v} \lesssim \epsilon$ and $E_{\kappa-1}^{d} \lesssim \epsilon$ yields

$$
X_{\kappa-1}^{d} \lesssim E_{\kappa-1}^{d}
$$

Furthermore, for $|a| \leq \kappa-1$, we get from (3.4) that

$$
X_{\kappa+1}^{d} \lesssim E_{\kappa+1}^{d}+E_{\kappa+1}^{d}\left(E_{\kappa-1}^{d}+E_{\kappa}^{v}\right)+\left(E_{\kappa-1}^{d}+E_{\kappa-1}^{v}\right) X_{\kappa+1}^{d}+\left(E_{\kappa+1}^{d}+E_{\kappa}^{v}\right) X_{\kappa-1}^{d},
$$

from which together with the assumption implies

$$
X_{\kappa+1}^{d} \lesssim E_{\kappa+1}^{d}
$$

Thus the lemma is proved.

An immediate consequence of the weighted $L^{2}$ norm estimate is that we can gain more decay for the good unknowns of the orientation field.

Lemma 3.3. Suppose $\kappa \geq 9, E_{\kappa}^{v} \lesssim \epsilon, E_{\kappa-1}^{d} \lesssim \epsilon$, then there hold

$$
\begin{gathered}
\left\|\langle t\rangle\left|\left(\partial_{t}+\partial_{r}\right) \partial Z^{a} d \|_{L^{2}} \lesssim\left(E_{\kappa-1}^{d}\right)^{\frac{1}{2}}, \quad \forall\right| a \mid \leq \kappa-3,\right. \\
\left\|\langle t\rangle\left|\left(\partial_{t}+\partial_{r}\right) \partial Z^{a} d \|_{L^{2}} \lesssim\left(E_{\kappa+1}^{d}\right)^{\frac{1}{2}}, \quad \forall\right| a \mid \leq \kappa-1 .\right.
\end{gathered}
$$

Proof. It is a direct consequence of Lemma 2.4. Lemma 3.1 and Lemma 3.2.

Another consequence is that we can obtain the decay for $L^{\infty}$ norm of $\partial Z^{a} d$.

Lemma 3.4. Suppose $\kappa \geq 9, E_{\kappa}^{v} \lesssim \epsilon, E_{\kappa-1}^{d} \lesssim \epsilon$, then there hold

$$
\begin{array}{ll}
\langle t\rangle\left\|\partial Z^{a} d\right\|_{L^{\infty}} \lesssim\left(E_{\kappa-1}^{d}\right)^{\frac{1}{2}}, & \forall|a| \leq \kappa-4, \\
\langle t\rangle\left\|\partial Z^{a} d\right\|_{L^{\infty}} \lesssim\left(E_{\kappa+1}^{d}\right)^{\frac{1}{2}}, & \forall|a| \leq \kappa-2 .
\end{array}
$$

Proof. By (2.18) and (2.19), one has

$$
\begin{aligned}
\left\|\partial Z^{a} d\right\|_{L^{\infty}} & \leq\langle t\rangle^{-1}\left(\left\|r \partial Z^{a} d\right\|_{L^{\infty}(r \geq\langle t\rangle / 2)}+\left\|\langle t\rangle \partial Z^{a} d\right\|_{L^{\infty}(r \leq\langle t\rangle / 2)}\right) \\
& \lesssim\langle t\rangle^{-1}\left[\left(E_{|a|+3}^{d}\right)^{\frac{1}{2}}+\left(X_{|a|+3}^{d}\right)^{\frac{1}{2}}\right] .
\end{aligned}
$$

Using Lemma 3.2 we can obtain the result. 


\section{Decay estimates for the velocity}

This section is devoted to the decay estimate under the a priori estimate assumption of the generalized energy. The estimate for the weighted $L^{2}$ norm in Section 3 is also applicable.

Lemma 4.1. Suppose $E_{\kappa}^{v}+\sum_{1 \leq|a| \leq \kappa}\left\|\nabla^{a} v\right\|_{L_{t, x}^{2}}+E_{\kappa-1}^{d} \lesssim \epsilon$ with $\kappa \geq 9$, then there hold

$$
\begin{aligned}
\left\|\langle t\rangle^{\frac{3}{4}} v\right\|_{L_{x}^{\infty}} & \lesssim \epsilon^{\frac{1}{2}} \\
\left\|\langle t\rangle^{\frac{5}{4}} \nabla v\right\|_{L_{x}^{\infty}} & \lesssim \epsilon^{\frac{1}{2}} \\
\left\|\langle t\rangle^{\frac{3}{2}} \nabla^{a} v\right\|_{L_{x}^{\infty}} & \lesssim \epsilon^{\frac{1}{2}}(\ln \langle t\rangle)^{\frac{1}{2}}, \quad \forall 2 \leq|a| \leq \kappa-3 .
\end{aligned}
$$

Remark 4.1. The first two decay estimates (4.1) and (4.2) are sharp in the sense that the decay rate is the same as the linear heat equation if the initial data lies in the energy space. The restricted decay rate for higher order derivatives (4.3) is due to the Ericksen stress.

Proof. Thanks to the local well-posedness [10, one easily has an uniform bound on the life span of lower-bound of $\delta^{-1}$ where $\delta$ is the size of the initial perturbation around equilibrium. Thus in the following argument, we always assume $t \geq 1$. Correspondingly, the $L_{t}^{p}$ norm denotes $L^{p}([1, t))$ for simplicity, where the integral time interval is $[1, t)$.

We begin by writing down the expression for velocity:

$$
v(t, x)=e^{t \Delta} v(1)-\int_{1}^{t} e^{(t-s) \Delta} \mathbb{P}[v \cdot \nabla v+\nabla \cdot(\nabla d \otimes \nabla d)](s) \mathrm{d} s,
$$

where $\mathbb{P}$ is the Leray projector. The inhomogeneous term can be rewritten as

$$
\begin{aligned}
& \int_{1}^{t} e^{(t-s) \Delta} \mathbb{P}[v \cdot \nabla v+\nabla \cdot(\nabla d \otimes \nabla d)](s) d s \\
& =\int_{1}^{t} \int_{\mathbb{R}^{3}} \nabla_{y} H(t-s, x-y)(v \otimes v+\nabla d \otimes \nabla d)(s, y) \mathrm{d} s \mathrm{~d} y,
\end{aligned}
$$

where $H(t, x)$ is a function of the three dimensional heat kernel $C t^{-\frac{3}{2}} \exp \left(-|x|^{2} / t\right)$ convoluting the Leray projection operator. Moreover, $H(t, x)$ behaves like (see for instance in [27, Proposition 11.1):

$$
t^{-\frac{3}{2}} h(|x| / \sqrt{t})
$$

where

$$
|h(y)| \lesssim 1 /\langle y\rangle^{3}, \quad\left|\nabla_{y} h(y)\right| \lesssim 1 /\langle y\rangle^{4}, \quad\left|\nabla_{y}^{2} h(y)\right| \lesssim 1 /\langle y\rangle^{5} .
$$

Now we are ready to show the lemma. We first prove (4.1). To this end, we write

$$
t^{\frac{3}{4}} v=J_{0}+J_{1}+J_{2}+J_{3},
$$

where

$$
\begin{aligned}
& J_{0}=t^{\frac{3}{4}} e^{t \Delta} v(1) \\
& J_{1}=t^{\frac{3}{4}} \int_{1}^{\frac{t}{2}} \int_{\mathbb{R}^{3}} \nabla_{y} H(t-s, x-y)(v \otimes v+\nabla d \otimes \nabla d)(s, y) \mathrm{d} s \mathrm{~d} y, \\
& J_{2}=t^{\frac{3}{4}} \int_{\frac{t}{2}}^{t} \int_{\mathbb{R}^{3}} \nabla_{y} H(t-s, x-y)(v \otimes v)(s, y) \mathrm{d} s \mathrm{~d} y \\
& J_{3}=t^{\frac{3}{4}} \int_{\frac{t}{2}}^{t} \int_{\mathbb{R}^{3}} \nabla_{y} H(t-s, x-y)(\nabla d \otimes \nabla d)(s, y) \mathrm{d} s \mathrm{~d} y .
\end{aligned}
$$


For $J_{0}$, one gets by Young's inequality that

$$
J_{0} \lesssim\|v(1, \cdot)\|_{L_{x}^{2}} \lesssim\left(E_{\kappa}^{v}\right)^{\frac{1}{2}}
$$

For $J_{1}$, one has $t / 2 \leq t-s \leq t$ from $1 \leq s \leq t / 2$. Hence applying Young's inequality and the Hardy-Littlewood-Sobolev inequality yields

$$
\begin{aligned}
J_{1} & \leq 2 \int_{1}^{\frac{t}{2}}(t-s)^{\frac{3}{4}}\|\nabla H(t-s, \cdot)\|_{L_{x}^{2}}\left\|\left(|v|^{2}+|\nabla d|^{2}\right)(s, \cdot)\right\|_{L_{x}^{2}} \mathrm{~d} s \\
& \lesssim \int_{1}^{\frac{t}{2}}(t-s)^{-\frac{1}{2}}\left\|\left(|v|^{2}+|\nabla d|^{2}\right)(s, \cdot)\right\|_{L_{x}^{2}} \mathrm{~d} s \\
& \lesssim\left\||v|^{2}+|\nabla d|^{2}\right\|_{L_{t, x}^{2}} .
\end{aligned}
$$

Then one can obtain the estimate for $J_{1}$ by using Lemma 3.4

$$
J_{1} \lesssim\|\nabla v\|_{L_{t, x}^{2}}\left(E_{\kappa}^{v}\right)^{\frac{1}{2}}+\left\|\langle t\rangle^{-1}\right\|_{L_{t}^{2}} E_{\kappa-1}^{d} \lesssim\|\nabla v\|_{L_{t, x}^{2}}^{2}+E_{\kappa}^{v}+E_{\kappa-1}^{d} .
$$

For the term $J_{2}$, one has $t / 2 \leq s \leq t$. Hence, by Young's inequality and the Hardy-Littlewood-Sobolev inequality, we can derive that

$$
\begin{aligned}
J_{2} & \leq \int_{\frac{t}{2}}^{t} s^{\frac{3}{4}}|\nabla H(t-s)| *|(v \otimes v)(s)| \mathrm{d} s \\
& \lesssim \int_{\frac{t}{2}}^{t}\|\nabla H(t-s)\|_{L_{x}^{1}}\|v(s)\|_{L_{x}^{\infty}}\left\|s^{\frac{3}{4}} v(s)\right\|_{L_{x}^{\infty}} \mathrm{d} s \\
& \lesssim \int_{\frac{t}{2}}^{t}(t-s)^{-\frac{1}{2}}\|v(s)\|_{L_{x}^{\infty}}\left\|s^{\frac{3}{4}} v(s)\right\|_{L_{x}^{\infty}} \mathrm{d} s \\
& \lesssim\|v(s)\|_{L_{t}^{2} L_{x}^{\infty}}\left\|s^{\frac{3}{4}} v(s)\right\|_{L_{t, x}^{\infty}} \\
& \lesssim \sum_{1 \leq|a| \leq 2}\left\|\nabla^{a} v\right\|_{L_{t}^{2} L_{x}^{2}}\left\|s^{\frac{3}{4}} v(s)\right\|_{L_{t, x}^{\infty}} .
\end{aligned}
$$

For $J_{3}$, similar to the estimate of $J_{2}$, one has

$$
J_{3} \lesssim\left\|s^{\frac{3}{4}} \nabla d(s)\right\|_{L_{t}^{\infty} L_{x}^{\infty}}\|\nabla d(s)\|_{L_{t}^{2} L_{x}^{\infty}} \lesssim E_{\kappa-1}^{d},
$$

where we used Lemma 3.4 in the last estimate. Gathering the estimates for $J_{0}, J_{1}, J_{2}, J_{3}$, we conclude

$$
t^{\frac{3}{4}} v(t) \lesssim\left(E_{\kappa}^{v}\right)^{\frac{1}{2}}+E_{\kappa}^{v}+E_{\kappa-1}^{d}+\|\nabla v\|_{L_{t, x}^{2}}^{2}+\sum_{1 \leq|a| \leq 2}\left\|\nabla^{a} v\right\|_{L_{t}^{2} L_{x}^{2}}\left\|s^{\frac{3}{4}} v(s)\right\|_{L_{t, x}^{\infty}} .
$$

Absorbing the last term yields (4.1).

Secondly, we treat (4.2). Similar to the estimate of (4.1), we write

$$
t^{\frac{5}{4}} \nabla v(t, x)=J_{0}^{1}+J_{1}^{1}+J_{2}^{1}+J_{3}^{1},
$$

where

$$
\begin{aligned}
& J_{0}^{1}=t^{\frac{5}{4}} \nabla e^{t \Delta} v(1) \\
& J_{1}^{1}=t^{\frac{5}{4}} \int_{1}^{\frac{t}{2}} \int_{\mathbb{R}^{3}} \nabla_{y}^{2} H(t-s, x-y)(v \otimes v+\nabla d \otimes \nabla d)(s, y) \mathrm{d} s \mathrm{~d} y, \\
& J_{2}^{1}=t^{\frac{5}{4}} \int_{\frac{t}{2}}^{t} \int_{\mathbb{R}^{3}} \nabla_{y} H(t-s, x-y) \nabla(v \otimes v)(s, y) \mathrm{d} s \mathrm{~d} y,
\end{aligned}
$$




$$
J_{3}^{1}=t^{\frac{5}{4}} \int_{\frac{t}{2}}^{t} \int_{\mathbb{R}^{3}} \nabla_{y} H(t-s, x-y) \nabla(\nabla d \otimes \nabla d)(s, y) \mathrm{d} s \mathrm{~d} y .
$$

We remark that the formulation of $J_{1}^{1}$ is different from $J_{1}$, while the other terms are similar.

For $J_{0}^{1}$, we get by Young's inequality that

$$
J_{0}^{1} \lesssim\|v(1, \cdot)\|_{L_{x}^{2}} \lesssim\left(E_{\kappa}^{v}\right)^{\frac{1}{2}} .
$$

Applying Young's inequality and the Hardy-Littlewood-Sobolev inequality, we have that

$$
\begin{aligned}
J_{1}^{1} & \leq 2 \int_{1}^{\frac{t}{2}}(t-s)^{\frac{5}{4}}\left\|\nabla^{2} H(t-s, \cdot)\right\|_{L_{x}^{2}}\left\|\left(|v|^{2}+|\nabla d|^{2}\right)(s, \cdot)\right\|_{L_{x}^{2}} \mathrm{~d} s \\
& \lesssim\left\||v|^{2}+|\nabla d|^{2}\right\|_{L_{t, x}^{2}} \lesssim\|\nabla v\|_{L_{t, x}^{2}}^{2}+E_{\kappa}^{v}+E_{\kappa-1}^{d},
\end{aligned}
$$

where the relation $t / 2 \leq t-s \leq t$ has been used. For $J_{2}^{1}$, we derive that

$$
J_{2}^{1} \lesssim \sum_{1 \leq|a| \leq 2}\left\|\nabla^{a} v\right\|_{L_{t}^{2} L_{x}^{2}}\left\|s^{\frac{5}{4}} \nabla v(s)\right\|_{L_{t, x}^{\infty}} .
$$

The estimate for $J_{3}^{1}$ is similar to $J_{2}$ with slight modifications. By Young's inequality, the HardyLittlewood-Sobolev inequality and Lemma 3.4, one derives that

$$
\begin{aligned}
J_{3}^{1} & \lesssim \int_{\frac{t}{2}}^{t}\|\nabla H(t-s)\|_{L_{x}^{1}}\left\|s^{\frac{1}{4}} \nabla^{2} d(s)\right\|_{L_{x}^{\infty}}\|s \nabla d(s)\|_{L_{x}^{\infty}} \mathrm{d} s \\
& \lesssim\left\|s^{-\frac{3}{4}}\right\|_{L_{t}^{2}}\left\|s \nabla^{2} d(s)\right\|_{L_{t, x}^{\infty}}\|s \nabla d(s)\|_{L_{t, x}^{\infty}} \lesssim E_{\kappa-1}^{d} .
\end{aligned}
$$

Gathering the estimate for $J_{0}^{1}, J_{1}^{1}, J_{2}^{1}, J_{3}^{1}$, we conclude

$$
t^{\frac{5}{4}} v(t) \lesssim\left(E_{\kappa}^{v}\right)^{\frac{1}{2}}+E_{\kappa}^{v}+E_{\kappa-1}^{d}+\|\nabla v\|_{L_{t, x}^{2}}^{2}+\sum_{1 \leq|a| \leq 2}\left\|\nabla^{a} v\right\|_{L_{t}^{2} L_{x}^{2}}\left\|s^{\frac{5}{4}} v(s)\right\|_{L_{t, x}^{\infty}} .
$$

Absorbing the last term yields (4.2).

Finally, we treat (4.3). For simplicity of presentation, we only show the case for $|a|=2$. The higher-order case can be estimated in the same but lengthier argument.

Similar to the estimate of (4.2), we write:

$$
t^{\frac{3}{2}} \nabla^{2} v(t, x)=J_{0}^{2}+J_{1}^{2}+J_{2}^{2}+J_{3}^{2},
$$

where

$$
\begin{aligned}
& J_{0}^{2}=t^{\frac{3}{2}} e^{t \Delta} \nabla^{2} v(1), \\
& J_{1}^{2}=t^{\frac{3}{2}} \int_{1}^{\frac{t}{2}} \int_{\mathbb{R}^{3}} \nabla_{y}^{2} H(t-s, x-y) \nabla(v \otimes v+\nabla d \otimes \nabla d)(s, y) \mathrm{d} s \mathrm{~d} y, \\
& J_{2}^{2}=t^{\frac{3}{2}} \int_{\frac{t}{2}}^{t} \int_{\mathbb{R}^{3}} \nabla_{y} H(t-s, x-y) \nabla^{2}(v \otimes v)(s, y) \mathrm{d} s \mathrm{~d} y, \\
& J_{3}^{2}=t^{\frac{3}{2}} \int_{\frac{t}{2}}^{t} \int_{\mathbb{R}^{3}} \nabla_{y} H(t-s, x-y) \nabla^{2}(\nabla d \otimes \nabla d)(s, y) \mathrm{d} s \mathrm{~d} y .
\end{aligned}
$$

As the estimates of $J_{0}^{2}, J_{1}^{2}$ and $J_{2}^{2}$ are similar to those of $J_{0}^{1}, J_{1}^{1}$ and $J_{2}^{1}$ respectively, we only sketch them here. By Young's inequality, the Hardy-Littlewood-Sobolev inequality and Lemma 3.4, one has

$$
J_{0}^{2} \lesssim t^{-\frac{1}{4}}\|v(1, \cdot)\|_{L_{x}^{2}} \lesssim\left(E_{\kappa}^{v}\right)^{\frac{1}{2}}
$$




$$
\begin{aligned}
J_{1}^{2} & \lesssim\|\nabla(v \otimes v+\nabla d \otimes \nabla d)\|_{L_{t}^{\frac{4}{3}} L_{x}^{2}} \lesssim \sum_{1 \leq|a| \leq \kappa}\left\|\nabla^{a} v\right\|_{L_{t, x}^{2}}^{2}+E_{\kappa}^{v}+E_{\kappa-1}^{d}, \\
J_{2}^{2} & \lesssim\left\|s^{\frac{3}{2}} \nabla^{2}(v \otimes v)\right\|_{L_{t}^{2} L_{x}^{\infty}} \\
& \lesssim \sum_{1 \leq|a| \leq 2}\left\|\nabla^{a} v\right\|_{L_{t}^{2} L_{x}^{2}}\left\|s^{\frac{3}{2}} \nabla^{2} v\right\|_{L_{t, x}^{\infty}}+\left\|s^{\frac{5}{4}} \nabla v\right\|_{L_{t, x}^{\infty}}^{2}\left\|s^{-1}\right\|_{L_{t}^{2}} .
\end{aligned}
$$

For $J_{3}^{2}$, by Young's inequality, the Hardy-Littlewood-Sobolev inequality, one derives that

$$
\begin{aligned}
J_{3}^{2} & \lesssim \int_{\frac{t}{2}}^{t}\left\|\nabla_{y} H(t-s)\right\|_{L_{x}^{p^{\prime}}} \sum_{2 \leq|a| \leq 3}\left\|s^{\frac{1}{2}} \nabla^{a} d(s)\right\|_{L_{x}^{p}} \sum_{1 \leq|a| \leq 2}\left\|s \nabla^{a} d(s)\right\|_{L_{x}^{\infty}} \mathrm{d} s \\
& \lesssim \int_{\frac{t}{2}}^{t}(t-s)^{-\frac{1}{2}-\frac{3}{2 p}} \sum_{2 \leq|a| \leq 3}\left\|s^{\frac{1}{2}} \nabla^{a} d(s)\right\|_{L_{x}^{p}} \sum_{1 \leq|a| \leq 2}\left\|s \nabla^{a} d(s)\right\|_{L_{x}^{\infty}} \mathrm{d} s \\
& \lesssim \sum_{2 \leq|a| \leq 3}\left\|s^{\frac{1}{2}} \nabla^{a} d(s)\right\|_{L_{t}^{\frac{2 p}{p-3}}} \sum_{L_{x}^{p}}\left\|s \nabla^{a} d(s)\right\|_{L_{t, x}^{\infty},},
\end{aligned}
$$

where $p$ and $p^{\prime}$ are dual index, $3<p \leq \infty$. Here, the constraint $3<p$ is due to the application of the Hardy-Littlewood-Sobolev inequality. To earn the maximum decay, we take $p=\infty$. Consequently, by Lemma 3.4, there holds

$$
J_{3}^{2} \lesssim\left(E_{\kappa-1}^{d}\right)^{\frac{1}{2}}\left\|s^{-\frac{1}{2}}\right\|_{L_{t}^{2}} \sum_{1 \leq|a| \leq 3}\left\|s \nabla^{a} d(s)\right\|_{L_{t, x}^{\infty}} \lesssim(\ln \langle t\rangle)^{\frac{1}{2}} E_{\kappa-1}^{d} .
$$

Finally, one conclude that

$$
\begin{aligned}
t^{\frac{3}{2}} \nabla^{2} v(t) \lesssim & \left(E_{\kappa}^{v}\right)^{\frac{1}{2}}+E_{\kappa}^{v}+(\ln \langle t\rangle)^{\frac{1}{2}} E_{\kappa-1}^{d}+\sum_{1 \leq|a| \leq \kappa}\left\|\nabla^{a} v\right\|_{L_{t, x}^{2}}^{2}+\left\|s^{\frac{5}{4}} \nabla v(s)\right\|_{L_{t, x}^{\infty}}^{2} \\
& +\sum_{1 \leq|a| \leq 2}\left\|\nabla^{a} v\right\|_{L_{t}^{2} L_{x}^{2}}\left\|s^{\frac{3}{2}} \nabla^{2} v\right\|_{L_{t, x}^{\infty}} .
\end{aligned}
$$

Absorbing the last term, (4.3) can be inferred from (4.2).

An immediate consequence of the above lemma is the decay estimate for $\partial_{t} v$.

Lemma 4.2. Under the assumption of Lemma 4.1, there holds

$$
\left\|\partial_{t} v\right\|_{L^{\infty}} \lesssim \epsilon^{\frac{1}{2}}\langle t\rangle^{-\frac{3}{2}}(\ln \langle t\rangle)^{\frac{1}{2}}
$$

Proof. We first show that (this decay rate is not optimal but it is sufficient for our purpose)

$$
\|\nabla p\|_{L^{\infty}} \lesssim \epsilon\langle t\rangle^{-\frac{5}{3}} .
$$

Due the incompressible condition for $v$, we can use the Leray projector to write the pressure explicitly:

$$
\nabla p=(\mathbb{P}-1)(v \nabla v+\nabla \cdot(\nabla d \otimes \nabla d)) .
$$

Then by Sobolev imbedding, one deduces that

$$
\begin{aligned}
\|\nabla p\|_{L^{\infty}} & \lesssim\|\nabla p\|_{L^{6}}^{\frac{1}{2}}\left\|\nabla^{2} p\right\|_{L^{6}}^{\frac{1}{2}} \\
& \lesssim \sum_{|a| \leq 1}\left\|\nabla^{a}(v \nabla v+\nabla \cdot(\nabla d \otimes \nabla d))\right\|_{L^{6}}
\end{aligned}
$$




$$
\begin{aligned}
\lesssim & \sum_{|a| \leq 1}\left(\left\|\nabla^{a} v\right\|_{L^{2}}^{\frac{1}{3}}\left\|\nabla^{a} v\right\|_{L^{\infty}}^{\frac{2}{3}}\right) \sum_{1 \leq|a| \leq 2}\left\|\nabla^{a} v\right\|_{L^{\infty}} \\
& +\sum_{|a| \leq 1}\left(\left\|\nabla \nabla^{a} d\right\|_{L^{2}}^{\frac{1}{3}}\left\|\nabla \nabla^{a} d\right\|_{L^{\infty}}^{\frac{2}{3}}\right) \sum_{1 \leq|a| \leq 2}\left\|\nabla \nabla^{a} d\right\|_{L^{\infty}} .
\end{aligned}
$$

all of which can be controlled by $\epsilon\langle t\rangle^{-\frac{5}{3}}$ by Lemma 3.4 and Lemma 4.1. Thus (4.5) is proved.

Then, thanks to Lemma 3.4. Lemma 4.1 and (4.5), we deduce that

$$
\begin{aligned}
\left\|\partial_{t} v\right\|_{L^{\infty}} & \lesssim\|\Delta v\|_{L^{\infty}}+\|v \cdot \nabla v\|_{L^{\infty}}+\|\nabla \cdot(\nabla d \otimes \nabla d)\|_{L^{\infty}}+\|\nabla p\|_{L^{\infty}} \\
& \lesssim\langle t\rangle^{-\frac{3}{2}}(\ln \langle t\rangle)^{\frac{1}{2}} \epsilon^{\frac{1}{2}},
\end{aligned}
$$

which yields the lemma.

\section{$5 \quad$ The energy estimates}

This section is devoted to the energy estimates, which corresponds to generalized energy estimate for the velocity, the higher-order and the lower-order generalized energy estimates for the orientation field.

\subsection{Generalized energy estimate for velocity}

In this subsection, we estimate the generalized energy for the velocity, which turns out to be uniformly bounded in time. The main trouble in the estimate of $E_{\kappa}^{v}$ is due to commutators between the scaling operator and the viscosity terms. Fortunately, we can take the approach borrowed from [3, 11.

Let $\kappa \geq 9,0 \leq|a| \leq \kappa$. Taking the $L^{2}$ inner product of $(2.6)$ with $Z^{a} v$, we have

$$
\frac{1}{2} \frac{d}{d t} \int_{\mathbb{R}^{n}}\left|Z^{a} v\right|^{2} \mathrm{~d} x-\int_{\mathbb{R}^{n}} \mu \Delta(S-1)^{a_{1}} \Gamma^{a^{\prime}} v \cdot Z^{a} v \mathrm{~d} x=\int_{\mathbb{R}^{n}} f_{a}^{1} \cdot Z^{a} v \mathrm{~d} x .
$$

Recalling the expression for $f_{a}^{1}$ in (2.7), one has

$$
\int_{\mathbb{R}^{n}} f_{\alpha a}^{1} \cdot Z^{a} v \mathrm{~d} x \lesssim \sum_{|b|+|c| \leq|a|}\left\|\nabla Z^{a} v\right\|_{L^{2}}\left(\left\|\left|\nabla Z^{b} d\right|\left|\nabla Z^{c} d\right|\right\|_{L^{2}}+\left\|\left|Z^{b} v\right|\left|Z^{c} v\right|\right\|_{L^{2}}\right) .
$$

Due to the symmetry between $b$ and $c$, we assume $|b| \leq|c|$ without loss of generality. Thus one has $|c| \leq[|a| / 2] \leq \kappa-4$. Consequently, by Lemma 3.4, the above can be further bounded by

$$
\begin{aligned}
& \left\|\nabla Z^{a} v\right\|_{L^{2}}\left(\left\|\nabla Z^{|a|} d\right\|_{L^{2}}\left\|\nabla Z^{[|a| / 2]} d\right\|_{L^{\infty}}+\left\|Z^{|a|} v\right\|_{L^{2}}\left\|Z^{[|a| / 2]} v\right\|_{L^{\infty}}\right) \\
& \lesssim\left\|\nabla Z^{a} v\right\|_{L^{2}}\left(\langle t\rangle^{-1}\left(E_{\kappa+1}^{d} E_{\kappa-1}^{d}\right)^{\frac{1}{2}}+\left\|\nabla Z^{\kappa} v\right\|_{L^{2}}\left\|Z^{\kappa} v\right\|_{L^{2}}\right) .
\end{aligned}
$$

Next, we estimate the diffusion terms with indefinite sign. To this end, we need a technical lemma followed from 3 .

Lemma 5.1. [3] (Iteration lemma) Let $\left\{f_{l}\right\},\left\{g_{l}\right\},\left\{F_{l}\right\}$ be three nonnegative sequences, where $0 \leq$ $l \leq \kappa$. Suppose that

$$
f_{0}+g_{0} \lesssim F_{0}
$$

and for all $1 \leq l \leq \kappa$,

$$
f_{l}+g_{l}-g_{l-1} \lesssim F_{l}
$$

Then there holds

$$
\sum_{0 \leq m \leq l}\left(f_{m}+g_{m}\right) \lesssim \sum_{0 \leq m \leq l} F_{m},
$$

for all $0 \leq l \leq \kappa$. 
Now we are ready to estimate the diffusion terms as follows:

$$
\begin{aligned}
& -\mu \int_{\mathbb{R}^{n}} \Delta(S-1)^{a_{1}} \Gamma^{a^{\prime}} v \cdot S^{a_{1}} \Gamma^{a^{\prime}} v \mathrm{~d} x \\
& =-\mu \sum_{l \leq a_{1}} C_{a_{1}}^{l}(-1)^{a_{1}-l} \int_{\mathbb{R}^{n}} \Delta S^{l} \Gamma^{a^{\prime}} v \cdot S^{a_{1}} \Gamma^{a^{\prime}} v \mathrm{~d} x \\
& \geq-\frac{1}{2} \mu\left\|\nabla S^{a_{1}} \Gamma^{a^{\prime}} v\right\|_{L^{2}}^{2}-\mu C \sum_{l \leq a_{1}-1}\left\|\nabla S^{l} \Gamma^{a^{\prime}} v\right\|_{L^{2}}^{2} .
\end{aligned}
$$

Inserting the above into (5.1), together with the estimates of the nonlinear terms yield

$$
\begin{aligned}
& \frac{1}{2} \frac{d}{d t} \int_{\mathbb{R}^{n}}\left|Z^{a} v\right|^{2} \mathrm{~d} x+\frac{1}{2} \mu\left\|\nabla Z^{a} v\right\|_{L_{x}^{2}}^{2}-\mu C \sum_{l \leq a_{1}-1}\left\|\nabla S^{l} \Gamma^{a^{\prime}} v\right\|_{L_{x}^{2}}^{2} \\
& \leq C\left\|\nabla Z^{\kappa} v\right\|_{L_{x}^{2}}^{2}\left\|Z^{\kappa} v\right\|_{L_{x}^{2}}^{2}+C\langle t\rangle^{-2} E_{\kappa+1}^{d} E_{\kappa-1}^{d} .
\end{aligned}
$$

Then integrating in time over $[0, t)$ on both sides of the above inequality gives

$$
\begin{aligned}
& \left\|Z^{a} v(t, \cdot)\right\|_{L_{x}^{2}}^{2}+\mu\left\|\nabla Z^{a} v\right\|_{L_{t}^{2} L_{x}^{2}}^{2}-\mu C \sum_{l \leq a_{1}-1}\left\|\nabla S^{l} \Gamma^{a^{\prime}} v\right\|_{L_{t}^{2} L_{x}^{2}}^{2} \\
& \lesssim\|Z v(0, \cdot)\|_{L_{x}^{2}}^{2}+\int_{0}^{t}\langle\tau\rangle^{-2} E_{\kappa+1}^{d}(\tau) E_{\kappa-1}^{d}(\tau) \mathrm{d} \tau+\left\|\nabla Z^{\kappa} v\right\|_{L_{t}^{2} L_{x}^{2}}^{2}\left\|Z^{\kappa} v\right\|_{L_{t}^{\infty} L_{x}^{2}}^{2} .
\end{aligned}
$$

Now we can use Lemma 5.1 to absorb the lower order diffusion terms to derive that

$$
\begin{aligned}
& \left\|Z^{\kappa} v(t, \cdot)\right\|_{L_{x}^{2}}^{2}+\mu\left\|\nabla Z^{\kappa} v\right\|_{L_{t}^{2} L_{x}^{2}}^{2} \\
& \lesssim\left\|Z^{\kappa} v(0, \cdot)\right\|_{L_{x}^{2}}^{2}+\int_{0}^{t}\langle\tau\rangle^{-2} E_{\kappa+1}^{d}(\tau) E_{\kappa-1}^{d}(\tau) \mathrm{d} \tau+\left\|\nabla Z^{\kappa} v(\cdot, \cdot)\right\|_{L_{t}^{2} L_{x}^{2}}^{2}\left\|Z^{\kappa} v(\cdot, \cdot)\right\|_{L_{t}^{\infty} L_{x}^{2}}^{2} .
\end{aligned}
$$

This gives the a priori estimate (2.9).

\subsection{Higher-order energy estimate for the orientation field}

In this subsection, we estimate the higher-order energy for the orientation field $d$, which will exhibit some polynomial growth in time. The main difficulty is the potential derivative loss due to the quasilinear effect. Fortunately, this difficulty can be overcome by a symmetry structure of the system. See the estimate of $I_{12}$ below.

Let $\kappa \geq 9,0 \leq|a| \leq \kappa$. Taking the $L^{2}$ inner product of $(2.6)_{2}$ with $\partial_{t} Z^{a} d$ gives

$$
\frac{1}{2} \frac{d}{d t} \int_{\mathbb{R}^{n}}\left|\partial Z^{a} d\right|^{2} \mathrm{~d} x=\int_{\mathbb{R}^{n}} f_{a}^{2} \cdot \partial_{t} Z^{a} d \mathrm{~d} x
$$

Thanks to (2.7), the right hand side above can be divided into $I_{1}$ and $I_{2}$, where

$$
\begin{aligned}
I_{1}= & -\int_{\mathbb{R}^{n}}\left[v \cdot \nabla\left(v \cdot \nabla Z^{a} d\right)+2 v \cdot \nabla \partial_{t} Z^{a} d\right] \cdot \partial_{t} Z^{a} d \mathrm{~d} x, \\
& -\int_{\mathbb{R}^{n}}\left(\partial_{t} Z^{a} v \cdot \nabla\right) d \cdot \partial_{t} Z^{a} d \mathrm{~d} x \\
\triangleq & I_{11}+I_{12}
\end{aligned}
$$

contains the highest order terms which may lose one derivative at first glance, and $I_{2}$ refers to the lower order ones:

$$
I_{2}=-\sum_{\substack{b+c=a \\ c \neq a}} C_{a}^{b} \int_{\mathbb{R}^{3}} 2 Z^{b} v \cdot \nabla \partial_{t} Z^{c} d \cdot \partial_{t} Z^{a} d \mathrm{~d} x
$$




$$
\begin{aligned}
& -\int_{\mathbb{R}^{3}} \sum_{\substack{++c=a \\
b \neq a}} C_{a}^{b}\left(\partial_{t} Z^{b} v \cdot \nabla Z^{c} d\right) \cdot \partial_{t} Z^{a} d \mathrm{~d} x \\
& +\int_{\mathbb{R}^{3}} \sum_{b+c+e=a} C_{a}^{b, c}\left(\nabla Z^{b} d \cdot \nabla Z^{c} d-\partial_{t} Z^{b} d \cdot \partial_{t} Z^{c} d\right) Z^{e} d \cdot \partial_{t} Z^{a} d \mathrm{~d} x \\
& -\int_{\mathbb{R}^{3}} \sum_{b+c+e+f=a} C_{a}^{b, c, e}\left[2\left(Z^{b} v \cdot \nabla\right) Z^{c} d \cdot \partial_{t} Z^{e} d\right] Z^{f} d \cdot \partial_{t} Z^{a} d \mathrm{~d} x \\
& -\int_{\mathbb{R}^{3}} \sum_{b+c+e+f+g=a} C_{a}^{b, c, e, f}\left[\left(Z^{b} v \cdot \nabla\right) Z^{c} d \cdot\left(\left(Z^{e} v \cdot \nabla\right) Z^{f} d\right)\right] Z^{g} d \cdot \partial_{t} Z^{a} d \mathrm{~d} x \\
& -\int_{\mathbb{R}^{3}} \sum_{\substack{b+c+e=a \\
e \neq a}} C_{a}^{b, c} Z^{b} v \cdot \nabla\left(Z^{c} v \cdot \nabla Z^{e} d\right) \cdot \partial_{t} Z^{a} d \mathrm{~d} x \\
& \triangleq I_{21}+I_{22}+I_{23}+I_{24}+I_{25}+I_{26} \text {. }
\end{aligned}
$$

Now we take care of the nonlinear terms group by group.

\subsubsection{Estimates of $I_{2}$}

\section{Estimate of $I_{21}$ :}

$$
I_{21}=-\sum_{\substack{b+c=a \\ c \neq a}} C_{a}^{b} \int_{\mathbb{R}^{3}} 2 Z^{b} v \cdot \nabla \partial_{t} Z^{c} d \cdot \partial_{t} Z^{a} d \mathrm{~d} x .
$$

If $|c| \leq|b|$, by Lemma 3.4, the above can be bounded by

$$
\sum_{\substack{b+c=a \\|c| \leq|b|}}\left\|Z^{b} v\right\|_{L^{2}}\left\|\nabla \partial_{t} Z^{c} d\right\|_{L^{\infty}}\left\|\partial_{t} Z^{a} d\right\|_{L^{2}} \lesssim\langle t\rangle^{-1}\left(E_{\kappa}^{v} E_{\kappa+1}^{d} E_{\kappa-1}^{d}\right)^{\frac{1}{2}} .
$$

Otherwise, if $|b| \leq|c|$, by (2.18) and Lemma 3.2, one has

$$
\begin{aligned}
& \sum_{\substack{|b| \leq|| a \mid / 2] \\
|c| \leq|a|-1}}\left(\int_{r \geq\langle t\rangle / 2}+\int_{r \leq\langle t\rangle / 2}\right)\left|Z^{b} v\right|\left|\nabla \partial_{t} Z^{c} d\right| \| \partial_{t} Z^{a} d \mid \mathrm{d} x \\
& \lesssim\langle t\rangle^{-1}\left\|r Z^{[|a| / 2]} v\right\|_{L^{\infty}(r \geq\langle t\rangle / 2)}\left\|\nabla \partial_{t} Z^{|a|-1} d\right\|_{L^{2}}\left\|\partial_{t} Z^{a} d\right\|_{L^{2}} \\
& +\langle t\rangle^{-1}\left\|Z^{[|a| / 2]} v\right\|_{L^{\infty}}\left\|\langle t-r\rangle \nabla \partial_{t} Z^{|a|-1} d\right\|_{L^{2}(r \leq\langle t\rangle / 2)}\left\|\partial_{t} Z^{a} d\right\|_{L^{2}} \\
& \lesssim\langle t\rangle^{-1}\left(E_{\kappa}^{v}\right)^{\frac{1}{2}} E_{\kappa+1}^{d}
\end{aligned}
$$

Estimate of $I_{22}$ :

$$
I_{22}=-\sum_{\substack{b+c=a \\ b \neq a}} C_{a}^{b} \int_{\mathbb{R}^{3}} \partial_{t} Z^{b} v \cdot \nabla Z^{c} d \cdot \partial_{t} Z^{a} d \mathrm{~d} x
$$

First for the case of $c=a$, the above quantity becomes

$$
-\int_{\mathbb{R}^{3}}\left(\partial_{t} v \cdot \nabla\right) Z^{a} d \cdot \partial_{t} Z^{a} d \mathrm{~d} x \lesssim\left\|\partial_{t} v\right\|_{L^{\infty}}\left\|\partial Z^{a} d\right\|_{L^{2}}^{2} .
$$

Next, if $|b| \leq|c| \leq|a|-1$, by (2.20), Lemma 3.2 and (2.18), one has

$$
-\sum_{\substack{b+c=a \\|b| \leq|c| \leq|a|-1}} C_{a}^{b}\left(\int_{r \leq\langle t\rangle / 2}+\int_{r \geq\langle t\rangle / 2}\right) \partial_{t} Z^{b} v \cdot \nabla Z^{c} d \cdot \partial_{t} Z^{a} d \mathrm{~d} x
$$




$$
\begin{aligned}
& \lesssim\langle t\rangle^{-1} \sum_{\substack{b+c=a \\
|b| \leq|c| \leq|a|-1}}\left\|\partial_{t} Z^{b} v\right\|_{L^{3}}\left\|\langle t-r\rangle \nabla Z^{c} d\right\|_{L^{6}(r \leq\langle t\rangle / 2)}\left\|\partial_{t} Z^{a} d\right\|_{L^{2}} \\
& \quad+\langle t\rangle^{-1} \sum_{\substack{b+c=a \\
|b| \leq|c| \leq|a|-1}}\left\|r \partial_{t} Z^{b} v\right\|_{L^{\infty}(r \geq\langle t\rangle / 2)}\left\|\nabla Z^{c} d\right\|_{L^{2}}\left\|\partial_{t} Z^{a} d\right\|_{L^{2}} \\
& \lesssim\langle t\rangle^{-1}\left(E_{\kappa}^{v}\right)^{\frac{1}{2}} E_{\kappa+1}^{d} .
\end{aligned}
$$

Otherwise, if $|c| \leq|b|$, by Lemma 3.4, we have the control of

$$
\left\|\partial_{t} Z^{|a|-1} v\right\|_{L^{2}}\left\|\nabla Z^{[|a| / 2]} d\right\|_{L^{\infty}}\left\|\partial_{t} Z^{a} d\right\|_{L^{2}} \lesssim\langle t\rangle^{-1}\left(E_{\kappa}^{v}\right)^{\frac{1}{2}} E_{\kappa+1}^{d} .
$$

\section{Estimate of $I_{23}$ :}

$$
\begin{aligned}
I_{23} & =\int_{\mathbb{R}^{3}} \sum_{b+c+e=a} C_{a}^{b, c}\left(\nabla Z^{b} d \cdot \nabla Z^{c} d-\partial_{t} Z^{b} d \cdot \partial_{t} Z^{c} d\right) Z^{e} d \cdot \partial_{t} Z^{a} d \mathrm{~d} x \\
& \lesssim \sum_{b+c+e=a} \int_{\mathbb{R}^{3}}\left|\partial Z^{b} d\right|\left|\partial Z^{c} d\right|\left|Z^{e} d\right|\left|\partial_{t} Z^{a} d\right| \mathrm{d} x .
\end{aligned}
$$

By Lemma 3.4, the above can be further bounded by

$$
\begin{aligned}
& \sum_{b+c+e=a} \int_{\mathbb{R}^{3}}\left|\partial Z^{b} d\right|\left|\partial Z^{c} d\right|\left|Z^{e} d \| \partial_{t} Z^{a} d\right| \mathrm{d} x \\
& \lesssim \sum_{[|a| / 2] \leq|e| \leq|a|}\left\|\partial Z^{[|a| / 2]} d\right\|_{L^{\infty}}\left\|\partial Z^{[|a| / 2]} d\right\|_{L^{3}}\left\|Z^{e} d\right\|_{L^{6}}\left\|\partial_{t} Z^{a} d\right\|_{L^{2}} \\
& \quad+\sum_{|e| \leq[|a| / 2]}\left\|\partial Z^{[|a| / 2]} d\right\|_{L^{\infty}}\left\|\partial Z^{|a|} d\right\|_{L^{2}}\left\|Z^{e} d\right\|_{L^{\infty}}\left\|\partial_{t} Z^{a} d\right\|_{L^{2}} \\
& \quad \lesssim\langle t\rangle^{-1} E_{\kappa+1}^{d} E_{\kappa-1}^{d} .
\end{aligned}
$$

\section{Estimate of $I_{24}$ :}

$$
\begin{aligned}
I_{24}= & -\int_{\mathbb{R}^{3}} \sum_{b+c+e+f=a} C_{a}^{b, c, e}\left[2\left(Z^{b} v \cdot \nabla\right) Z^{c} d \cdot \partial_{t} Z^{e} d\right] Z^{f} d \cdot \partial_{t} Z^{a} d \mathrm{~d} x \\
& \lesssim \int_{\mathbb{R}^{3}} \sum_{b+c+e+f=a}\left|Z^{b} v\right|\left|\partial Z^{c} d\right|\left|\partial Z^{e} d\right|\left|Z^{f} d\right|\left|\partial_{t} Z^{a} d\right| \mathrm{d} x .
\end{aligned}
$$

If $|f| \geq[|a| / 2]$, by Sobolev inequalities and Lemma 3.4, the above can controlled by

$$
\begin{aligned}
& \sum_{[|a| / 2] \leq|f| \leq|a|}\left\|Z^{[|a| / 2]} v\right\|_{L^{\infty}}\left\|\partial Z^{[|a| / 2]} d\right\|_{L^{\infty}}\left\|\partial Z^{[|a| / 2]} d\right\|_{L^{3}}\left\|Z^{f} d\right\|_{L^{6}}\left\|\partial_{t} Z^{a} d\right\|_{L^{2}} \\
& \lesssim\langle t\rangle^{-\frac{3}{2}}\left\|\nabla Z^{\kappa-1} v\right\|_{L^{2}} E_{\kappa+1}^{d} E_{\kappa-1}^{d} .
\end{aligned}
$$

Otherwise, if $|f| \leq[|a| / 2]$, by Lemma 3.4, $I_{34}$ can be controlled by

$$
\begin{aligned}
& \sum_{|f| \leq[|a| / 2]}\left\|Z^{|a| / 2} v\right\|_{L^{\infty}}\left\|\partial Z^{|a|} d\right\|_{L^{2}}\left\|\partial Z^{[|a| / 2]} d\right\|_{L^{\infty}}\left\|Z^{f} d\right\|_{L^{\infty}}\left\|\partial_{t} Z^{a} d\right\|_{L^{2}} \\
& +\sum_{|f| \leq[|a| / 2]}\left\|Z^{|a|} v\right\|_{L^{2}}\left\|\partial Z^{[a / 2]} d\right\|_{L^{\infty}}\left\|\partial Z^{[|a| / 2]} d\right\|_{L^{\infty}}\left\|Z^{f} d\right\|_{L^{\infty}}\left\|\partial_{t} Z^{a} d\right\|_{L^{2}} \\
& \lesssim\langle t\rangle^{-1} E_{\kappa+1}^{d}\left(E_{\kappa}^{v} E_{\kappa-1}^{d}\right)^{\frac{1}{2}}\left[1+\left(E_{\kappa-1}^{d}\right)^{\frac{1}{2}}\right] .
\end{aligned}
$$


Estimate of $I_{25}$ : By Lemma 3.4 we get

$$
\begin{aligned}
I_{25}= & -\sum_{b+c+e+f+g=a} C_{a}^{b, c, e, f} \int_{\mathbb{R}^{3}}\left[\left(Z^{b} v \cdot \nabla\right) Z^{c} d \cdot\left(\left(Z^{e} v \cdot \nabla\right) Z^{f} d\right)\right] Z^{g} d \cdot \partial_{t} Z^{a} d \mathrm{~d} x \\
\lesssim & \sum_{[|a| / 2] \leq|g| \leq|a|}\left\|Z^{[|a| / 2]} v\right\|_{L^{6}}^{2}\left\|Z^{[|a| / 2]} d\right\|_{L^{\infty}}^{2}\left\|Z^{g} d\right\|_{L^{6}}\left\|\partial_{t} Z^{a} d\right\|_{L^{2}} \\
& +\sum_{|g| \leq[|a| / 2]}\left(\left\|Z^{|a|} v\right\|_{L^{2}}\left\|Z^{[|a| / 2]} v\right\|_{L^{\infty}}\left\|\nabla Z^{[|a| / 2]} d\right\|_{L^{\infty}}^{2}\right. \\
& \left.\quad+\left\|Z^{[|a| / 2]} v\right\|_{L^{\infty}}^{2}\left\|\nabla Z^{|a|} d\right\|_{L^{2}}\left\|\nabla Z^{[|a| / 2]} d\right\|_{L^{\infty}}\right)\left\|Z^{g} d\right\|_{L^{\infty}}\left\|\partial_{t} Z^{a} d\right\|_{L^{2}} \\
& \lesssim\langle t\rangle^{-1}\left\|\nabla Z^{\kappa-1} v\right\|_{L^{2}}\left(E_{\kappa}^{v}\right)^{\frac{1}{2}} E_{\kappa+1}^{d}\left(1+\left(E_{\kappa-1}^{d}\right)^{\frac{1}{2}}\right) .
\end{aligned}
$$

Estimate of $I_{26}$ : By the Hölder inequality, one has

$$
I_{26}=-\sum_{\substack{b+c+e=a \\ e \neq a}} C_{a}^{b, c} \int_{\mathbb{R}^{3}} Z^{b} v \cdot \nabla\left(Z^{c} v \cdot \nabla Z^{e} d\right) \cdot \partial_{t} Z^{a} d \mathrm{~d} x \lesssim\left\|\nabla Z^{\kappa} v\right\|_{L^{2}}^{2} E_{\kappa+1}^{d} .
$$

\subsubsection{Estimate of $I_{1}$}

We estimate $I_{1}$ in this part.

Estimate of $I_{11}$ : Employing integration by parts, one has

$$
\begin{aligned}
I_{11} & =-\int_{\mathbb{R}^{n}}\left[v \cdot \nabla\left(v \cdot \nabla Z^{a} d\right)\right] \cdot \partial_{t} Z^{a} d \mathrm{~d} x \\
& =-\int_{\mathbb{R}^{n}}\left(\partial_{t} v \cdot \nabla\right) Z^{a} d \cdot(v \cdot \nabla) Z^{a} d \mathrm{~d} x+\frac{1}{2} \partial_{t} \int_{\mathbb{R}^{n}}\left|(v \cdot \nabla) Z^{a} d\right|^{2} \mathrm{~d} x \\
& \leq\left\|\nabla Z^{\kappa-1} v\right\|_{L_{x}^{2}}^{2} E_{\kappa+1}^{d}+\frac{1}{2} \partial_{t} \int_{\mathbb{R}^{n}}\left|(v \cdot \nabla) Z^{a} d\right|^{2} \mathrm{~d} x .
\end{aligned}
$$

Estimate of $I_{12}$ : The difficulty in estimating $I_{12}$ lies on the possible derivative loss problem. However, this difficulty can be bypassed by using the symmetry structure of the system.

Employing integration by parts, we have

$$
\begin{aligned}
I_{12}= & -\int_{\mathbb{R}^{n}}\left(\partial_{t} Z^{a} v \cdot \nabla\right) d \cdot \partial_{t} Z^{a} d \mathrm{~d} x \\
= & -\partial_{t} \int_{\mathbb{R}^{n}}\left(Z^{a} v \cdot \nabla\right) d \cdot \partial_{t} Z^{a} d \mathrm{~d} x+\int_{\mathbb{R}^{n}}\left(Z^{a} v \cdot \nabla\right) \partial_{t} d \cdot \partial_{t} Z^{a} d \mathrm{~d} x \\
& +\int_{\mathbb{R}^{n}}\left(Z^{a} v \cdot \nabla\right) d \cdot \partial_{t t} Z^{a} d \mathrm{~d} x .
\end{aligned}
$$

By Lemma 3.4, the second term on the right hand side of (5.2) is controlled by

$$
\left\|Z^{a} v\right\|_{L^{2}}\left\|\partial_{t} Z^{a} d\right\|_{L^{2}}\left\|\nabla \partial_{t} d\right\|_{L^{\infty}} \lesssim\langle t\rangle^{-1}\left(E_{\kappa}^{v} E_{\kappa+1}^{d} E_{\kappa-1}^{d}\right)^{\frac{1}{2}} .
$$

For the last term of (5.2), we are going to insert the equation $(2.6){ }_{2}$ for the orientation field into this expression to show the symmetry:

$$
\begin{aligned}
& \int_{\mathbb{R}^{n}}\left(Z^{a} v \cdot \nabla\right) d \cdot \partial_{t t} Z^{a} d \mathrm{~d} x \\
& =\int_{\mathbb{R}^{n}}\left(Z^{a} v \cdot \nabla\right) d \cdot \Delta Z^{a} d \mathrm{~d} x+\int_{\mathbb{R}^{n}}\left(Z^{a} v \cdot \nabla\right) d \cdot f_{a}^{2} \mathrm{~d} x .
\end{aligned}
$$


Thanks to Lemma 4.5, we get by integration by parts that

$$
\begin{aligned}
\int_{\mathbb{R}^{n}}\left(Z^{a} v \cdot \nabla\right) d \cdot \Delta Z^{a} d \mathrm{~d} x & \leq\left\|\nabla Z^{a} d\right\|_{L^{2}}\left(\left\|\nabla Z^{a} v\right\|_{L^{2}}\|\nabla d\|_{L^{\infty}}+\left\|Z^{a} v\right\|_{L^{2}}\left\|\nabla^{2} d\right\|_{L^{\infty}}\right) \\
& \lesssim\langle t\rangle^{-1}\left(E_{\kappa+1}^{d} E_{\kappa-1}^{d}\right)^{\frac{1}{2}}\left(\left\|\nabla Z^{a} v\right\|_{L^{2}}+\left(E_{\kappa}^{v}\right)^{\frac{1}{2}}\right) .
\end{aligned}
$$

On the other hand, it holds that

\section{Lemma 5.2.}

$$
\begin{aligned}
\int_{\mathbb{R}^{n}}\left(Z^{a} v \cdot \nabla\right) d \cdot f_{a}^{2} \mathrm{~d} x \leq & -\frac{1}{2} \frac{d}{d t} \int_{\mathbb{R}^{n}}\left|\left(Z^{a} v \cdot \nabla\right) d\right|^{2} \mathrm{~d} x+\langle t\rangle^{-1}\left(E_{\kappa}^{v}+E_{\kappa-1}^{d}\right)^{\frac{1}{2}} E_{\kappa+1}^{d} \\
& +\left\|\nabla Z^{\kappa} v\right\|_{L^{2}}^{2} E_{\kappa+1}^{d}+\langle t\rangle^{-1}\left\|\nabla Z^{\kappa} v\right\|_{L^{2}} E_{\kappa+1}^{d}+\left\|\partial_{t} v\right\|_{L^{\infty}} E_{\kappa+1}^{d} .
\end{aligned}
$$

Proof. Inserting the expression of $f_{a}^{2}$ in (2.7) into $\int_{\mathbb{R}^{n}}\left(Z^{a} v \cdot \nabla\right) d \cdot f_{a}^{2} \mathrm{~d} x$, we get

$$
\begin{aligned}
& \int_{\mathbb{R}^{n}}\left(Z^{a} v \cdot \nabla\right) d \cdot f_{a}^{2} \mathrm{~d} x \\
& =-\int_{\mathbb{R}^{n}}\left(Z^{a} v \cdot \nabla\right) d \cdot\left[(v \cdot \nabla)\left(v \cdot \nabla Z^{a} d+2 \partial_{t} Z^{a} d\right)+\partial_{t} Z^{a} v \cdot \nabla d\right] \mathrm{d} x+L,
\end{aligned}
$$

where

$$
\begin{aligned}
L= & \sum_{b+c+e=a} C_{a}^{b, c} \int_{\mathbb{R}^{n}}\left(Z^{a} v \cdot \nabla\right) d \cdot\left(\nabla Z^{b} d \cdot \nabla Z^{c} d-\partial_{t} Z^{b} d \cdot \partial_{t} Z^{c} d\right) Z^{e} d \mathrm{~d} x \\
& -\sum_{b+c+e+f=a} C_{a}^{b, c, e} \int_{\mathbb{R}^{n}}\left(Z^{a} v \cdot \nabla\right) d \cdot\left[2\left(Z^{b} v \cdot \nabla\right) Z^{c} d \cdot \partial_{t} Z^{e} d\right] Z^{f} d \mathrm{~d} x \\
& -\sum_{\substack{b+c+e+f+g=a\\
}} C_{a}^{b, c, e, f} \int_{\mathbb{R}^{n}}\left(Z^{a} v \cdot \nabla\right) d \cdot\left[\left(Z^{b} v \cdot \nabla\right) Z^{c} d \cdot\left(\left(Z^{e} v \cdot \nabla\right) Z^{f} d\right)\right] Z^{g} d \mathrm{~d} x \\
& -\sum_{\substack{b+c+e=a \\
e \neq a}} C_{a}^{b, c} \int_{\mathbb{R}^{n}}\left(Z^{a} v \cdot \nabla\right) d \cdot Z^{b} v \cdot \nabla\left(Z^{c} v \cdot \nabla Z^{e} d\right) \mathrm{d} x \\
& -\sum_{\substack{b+c=a \\
c \neq a}} C_{a}^{b} \int_{\mathbb{R}^{n}}\left(Z^{a} v \cdot \nabla\right) d \cdot\left(2 Z^{b} v \cdot \nabla \partial_{t} Z^{c} d+\partial_{t} Z^{c} v \cdot \nabla Z^{b} d\right) \mathrm{d} x \\
\triangleq & K_{1}+K_{2}+K_{3}+K_{4}+K_{5} .
\end{aligned}
$$

The first term on the right hand side of (5.4) refers to the highest order term, while the remaining terms denoted by $L$ refers to the lower order ones. The estimate of $L$ is similar but much easier than that of $I_{2}$, because $I_{2}$ contains quadratic terms, while $L$ contains only cubic terms or higher.

Now let us estimate the right hand side of (5.4) one by one. The first part of the higher order terms can be bounded by

$$
\begin{aligned}
& -\int_{\mathbb{R}^{n}}\left(Z^{a} v \cdot \nabla\right) d \cdot\left[(v \cdot \nabla)\left(v \cdot \nabla Z^{a} d+2 \partial_{t} Z^{a} d\right)\right] \mathrm{d} x \\
& \lesssim\left\|\nabla Z^{a} d\right\|_{L^{2}}\|v\|_{L^{\infty}}^{2}\left(\left\|\nabla Z^{a} v\right\|_{L^{2}}\|\nabla d\|_{L^{\infty}}+\left\|Z^{a} v\right\|_{L^{2}}\left\|\nabla^{2} d\right\|_{L^{\infty}}\right) \\
& +\left\|\partial_{t} Z^{a} d\right\|_{L^{2}}\|v\|_{L^{\infty}}\left\|\nabla Z^{a} v\right\|_{L^{2}}\|\nabla d\|_{L^{\infty}}+\left\|\partial_{t} Z^{a} d\right\|_{L^{2}}\|v\|_{L^{6}}\left\|Z^{a} v\right\|_{L^{6}}\left\|\nabla^{2} d\right\|_{L^{6}} \\
& \lesssim E_{\kappa+1}^{d}\left(\left(E_{\kappa}^{v}\right)^{\frac{1}{2}}+1\right)\left\|\nabla Z^{\kappa} v\right\|_{L^{2}}^{2} .
\end{aligned}
$$


For the second part of the higher order terms, now one can see the symmetry is present. Thus, employing integration by parts, one has

$$
\begin{aligned}
& -\int_{\mathbb{R}^{n}}\left(Z^{a} v \cdot \nabla\right) d \cdot\left(\partial_{t} Z^{a} v \cdot \nabla d\right) \mathrm{d} x \\
& =-\frac{1}{2} \frac{d}{d t} \int_{\mathbb{R}^{n}}\left|\left(Z^{a} v \cdot \nabla\right) d\right|^{2} \mathrm{~d} x+\int_{\mathbb{R}^{n}}\left(Z^{a} v \cdot \partial_{t} \nabla\right) d \cdot\left(Z^{a} v \cdot \nabla d\right) \mathrm{d} x \\
& \leq-\frac{1}{2} \frac{d}{d t} \int_{\mathbb{R}^{n}}\left|\left(Z^{a} v \cdot \nabla\right) d\right|^{2} \mathrm{~d} x+\left\|\nabla Z^{\kappa} v\right\|_{L^{2}}^{2} E_{\kappa+1}^{d} .
\end{aligned}
$$

Now we show the estimate for $L$ in (5.4). The estimate is similar to the estimates of $I_{1}$ to $I_{6}$ in the higher-order energy estimate for the orientation field. Hence we only sketch the argument. Due to Hölder inequality, (2.18), (2.19), (2.19), Lemma 3.2 and Lemma 3.4, one deduces that

$$
\begin{aligned}
K_{1} \lesssim & \left\|Z^{a} v\right\|_{L^{6}}\|\nabla d\|_{L^{\infty}}\left\|\partial Z^{|a|} d\right\|_{L^{2}}\left\|\partial Z^{[|a| / 2]} d\right\|_{L^{3}}\left\|Z^{[|a| / 2]} d\right\|_{L^{\infty}} \\
& +\left\|Z^{a} v\right\|_{L^{6}}\|\nabla d\|_{L^{\infty}}\left\|\partial Z^{[|a| / 2]} d\right\|_{L^{2}}\left\|\partial Z^{[|a| / 2]} d\right\|_{L^{6}}\left\|Z^{|a|} d\right\|_{L^{6}} \\
\lesssim & \langle t\rangle^{-1}\left\|\nabla Z^{\kappa} v\right\|_{L^{2}}\left(E_{\kappa+1}^{d}\right)^{\frac{1}{2}} E_{\kappa-1}^{d}\left(1+\left(E_{\kappa-1}^{d}\right)^{\frac{1}{2}}\right)
\end{aligned}
$$

and

$$
\begin{aligned}
K_{2} \lesssim & \left\|Z^{a} v\right\|_{L^{6}}\|\nabla d\|_{L^{\infty}}\left\|Z^{|a|} v\right\|_{L^{6}}\left(\left\|\partial Z^{|a|} d\right\|_{L^{2}}\left\|\partial Z^{[|a| / 2]} d\right\|_{L^{6}}\left\|Z^{[|a| / 2]} d\right\|_{L^{\infty}}\right. \\
& \left.+\left\|\partial Z^{[|a| / 2]} d\right\|_{L^{2}}\left\|\partial Z^{[|a| / 2]} d\right\|_{L^{\infty}}\left\|Z^{|a|} d\right\|_{L^{6}}\right) \\
\lesssim & \langle t\rangle^{-1}\left\|\nabla Z^{\kappa} v\right\|_{L^{2}}^{2}\left(E_{\kappa+1}^{d}\right)^{\frac{1}{2}} E_{\kappa-1}^{d}\left(1+\left(E_{\kappa-1}^{d}\right)^{\frac{1}{2}}\right),
\end{aligned}
$$

and

$$
\begin{aligned}
K_{3} \lesssim & \left\|Z^{a} v\right\|_{L^{6}}\|\nabla d\|_{L^{\infty}}\left\|Z^{|a|} v\right\|_{L^{6}}\left(\left\|\nabla Z^{|a|} d\right\|_{L^{2}}\left\|Z^{[|a| / 2]} v\right\|_{L^{\infty}}\left\|\nabla Z^{[|a| / 2]} d\right\|_{L^{6}}\left\|Z^{[|a| / 2]} d\right\|_{L^{\infty}}\right. \\
& +\left\|\nabla Z^{[|a| / 2]} d\right\|_{L^{\infty}}\left\|Z^{|a|} v\right\|_{L^{2}}\left\|\nabla Z^{[|a| / 2]} d\right\|_{L^{6}}\left\|Z^{[|a| / 2]} d\right\|_{L^{\infty}} \\
& \left.+\left\|\nabla Z^{[|a| / 2]} v\right\|_{L^{2}}\left\|\nabla Z^{[|a| / 2]} d\right\|_{L^{\infty}}^{2}\left\|Z^{|a|} d\right\|_{L^{6}}\right) \\
\lesssim & \langle t\rangle^{-1}\left\|\nabla Z^{\kappa} v\right\|_{L^{2}}^{2}\left(E_{\kappa+1}^{d} E_{\kappa}^{v}\right)^{\frac{1}{2}} E_{\kappa-1}^{d}\left(1+\left(E_{\kappa-1}^{d}\right)^{\frac{1}{2}}\right),
\end{aligned}
$$

and

$$
\begin{aligned}
K_{4} \lesssim & \left\|Z^{a} v\right\|_{L^{6}}\|\nabla d\|_{L^{\infty}}\left\|Z^{|a|} v\right\|_{L^{6}}\left\|Z^{[|a| / 2]+1} v\right\|_{L^{6}}\left\|\nabla Z^{[|a| / 2]+1} d\right\|_{L^{2}} \\
& +\left\|Z^{a} v\right\|_{L^{6}}\|\nabla d\|_{L^{\infty}}\left\|Z^{[|a| / 2]} v\right\|_{L^{3}}\left(\left\|\nabla Z^{|a|} v\right\|_{L^{2}}\left\|\nabla Z^{[|a| / 2]} d\right\|_{L^{\infty}}\right. \\
& \left.+\left\|Z^{[|a| / 2]+1} v\right\|_{L^{\infty}}\left\|\nabla Z^{|a|} d\right\|_{L^{2}}+\left\|Z^{|a|} v\right\|_{L^{6}}\left\|\nabla^{2} Z^{[|a| / 2]} d\right\|_{L^{3}}\right) \\
\lesssim & \langle t\rangle^{-1}\left\|\nabla Z^{\kappa}\right\|_{L^{2}}^{2}\left(E_{\kappa}^{v} E_{\kappa-1}^{d} E_{\kappa+1}^{d}\right)^{\frac{1}{2}}
\end{aligned}
$$

and

$$
K_{5} \lesssim\left\|Z^{a} v\right\|_{L^{6}}\|\nabla d\|_{L^{6}}\left\|Z^{|a|} v\right\|_{L^{6}}\left\|\nabla Z^{|a|} d\right\|_{L^{2}} \lesssim\left\|\nabla Z^{\kappa} v\right\|_{L^{2}}^{2} E_{\kappa+1}^{d}
$$

Combining all the above estimates and noting $E_{\kappa}^{v} \lesssim \epsilon$ and $E_{\kappa-1}^{d} \lesssim \epsilon$, we get the lemma.

\subsubsection{Completing the estimates}

Combining all the above estimates in this subsection, one has

$$
\frac{1}{2} \frac{d}{d t} \int_{\mathbb{R}^{n}}\left|\partial Z^{a} d\right|^{2} \mathrm{~d} x-\frac{1}{2} \frac{d}{d t} \int_{\mathbb{R}^{n}}\left|(v \cdot \nabla) Z^{a} d\right|^{2} \mathrm{~d} x
$$




$$
\begin{aligned}
& +\frac{d}{d t} \int_{\mathbb{R}^{n}}\left(Z^{a} v \cdot \nabla\right) d \cdot \partial_{t} Z^{a} d \mathrm{~d} x+\frac{1}{2} \frac{d}{d t} \int_{\mathbb{R}^{n}}\left|\left(Z^{a} v \cdot \nabla\right) d\right|^{2} \mathrm{~d} x \\
\lesssim & \langle t\rangle^{-1}\left(E_{\kappa}^{v}+E_{\kappa-1}^{d}\right)^{\frac{1}{2}} E_{\kappa+1}^{d}+\left\|\nabla Z^{\kappa} v\right\|_{L^{2}}^{2} E_{\kappa+1}^{d} \\
& +\langle t\rangle^{-1}\left\|\nabla Z^{\kappa} v\right\|_{L^{2}} E_{\kappa+1}^{d}+\left\|\partial_{t} v\right\|_{L^{\infty}} E_{\kappa+1}^{d} .
\end{aligned}
$$

Summing over $|a| \leq \kappa$, and noting that

$$
\sum_{|a| \leq \kappa} \int_{\mathbb{R}^{n}}\left|\left(Z^{a} v \cdot \nabla\right) d\right|^{2} \mathrm{~d} x \lesssim E_{\kappa}^{v} E_{\kappa-1}^{d} \lesssim \varepsilon E_{\kappa-1}^{d},
$$

we can deduce

$$
\begin{aligned}
& \sum_{|a| \leq \kappa}\left(\frac{1}{2} \int_{\mathbb{R}^{n}}\left|\partial Z^{a} d\right|^{2} \mathrm{~d} x-\frac{1}{2} \int_{\mathbb{R}^{n}}\left|(v \cdot \nabla) Z^{a} d\right|^{2} \mathrm{~d} x\right. \\
& \left.\quad+\int_{\mathbb{R}^{n}}\left(Z^{a} v \cdot \nabla\right) d \cdot \partial_{t} Z^{a} d \mathrm{~d} x+\frac{1}{2} \int_{\mathbb{R}^{n}}\left|\left(Z^{a} v \cdot \nabla\right) d\right|^{2} \mathrm{~d} x\right) \\
& \sim \frac{1}{2} \sum_{|a| \leq \kappa} \int_{\mathbb{R}^{n}}\left|\partial Z^{a} d\right|^{2} \mathrm{~d} x=\frac{1}{2} E_{\kappa+1}^{d} .
\end{aligned}
$$

Here we have used the assumption $E_{\kappa}^{v} \lesssim \epsilon$ and $E_{\kappa-1}^{d} \lesssim \epsilon$ again. This gives (2.10).

\subsection{Lower-order energy estimate for the orientation field}

This subsection is devoted to the lower-order energy estimate for the orientation field $d$. It turns out that the lower-order energy is uniformly bounded. To this end, we need to obtain the subcritical decay for the nonlinearities or say, $L^{1}$ integrability in time.

Let $\kappa \geq 9,0 \leq|a| \leq \kappa-2$. Taking the $L^{2}$ inner product of $(2.6)_{2}$ with $\partial_{t} Z^{a} d$, we have

$$
\frac{1}{2} \frac{d}{d t} \int_{\mathbb{R}^{n}}\left|\partial Z^{a} d\right|^{2} \mathrm{~d} x=\int_{\mathbb{R}^{n}} f_{a}^{2} \cdot \partial_{t} Z^{a} d \mathrm{~d} x
$$

Recalling the expression of $f_{a}^{2}$ in (2.7), we will rewrite the right hand side of the above equality as

$$
\begin{aligned}
& -\sum_{b+c=a} C_{a}^{b} \int_{\mathbb{R}^{3}}\left(2 Z^{b} v \cdot \nabla \partial_{t} Z^{c} d+\partial_{t} Z^{b} v \cdot \nabla Z^{c} d\right) \cdot \partial_{t} Z^{a} d \mathrm{~d} x \\
& +\int_{\mathbb{R}^{3}} \sum_{b+c+e=a} C_{a}^{b, c}\left(\nabla Z^{b} d \cdot \nabla Z^{c} d-\partial_{t} Z^{b} d \cdot \partial_{t} Z^{c} d\right) Z^{e} d \cdot \partial_{t} Z^{a} d \mathrm{~d} x \\
& -\sum_{b+c+e=a} C_{a}^{b, c} \int_{\mathbb{R}^{3}} Z^{b} v \cdot \nabla\left(Z^{c} v \cdot \nabla Z^{e} d\right) \cdot \partial_{t} Z^{a} d \mathrm{~d} x \\
& -\sum_{b+c+e+f=a} C_{a}^{b, c, e} \int_{\mathbb{R}^{3}}\left[2\left(Z^{b} v \cdot \nabla\right) Z^{c} d \cdot \partial_{t} Z^{e} d\right] Z^{f} d \cdot \partial_{t} Z^{a} d \mathrm{~d} x \\
& -\sum_{b+c+e+f+g=a} C_{a}^{b, c, e, f} \int_{\mathbb{R}^{3}}\left[\left(Z^{b} v \cdot \nabla\right) Z^{c} d \cdot\left(\left(Z^{e} v \cdot \nabla\right) Z^{f} d\right)\right] Z^{g} d \cdot \partial_{t} Z^{a} d \mathrm{~d} x \\
& =M_{1}+M_{2}+M_{3}+M_{4}+M_{5} .
\end{aligned}
$$

In the sequel, we will estimate $M_{1}$ to $M_{5}$ one by one.

We first estimate $M_{1}$. There are two terms inside the expression for $M_{1}$. In order to estimate them in a uniform way, we write by integration by parts that

$$
\sum_{b+c=a} C_{a}^{b} \int_{\mathbb{R}^{3}}\left(\partial_{t} Z^{b} v \cdot \nabla Z^{c} d\right) \cdot \partial_{t} Z^{a} d \mathrm{~d} x=\sum_{b+c=a} C_{a}^{b} \partial_{t} \int_{\mathbb{R}^{3}}\left(Z^{b} v \cdot \nabla Z^{c} d\right) \cdot \partial_{t} Z^{a} d \mathrm{~d} x
$$




$$
-\sum_{b+c=a} C_{a}^{b} \int_{\mathbb{R}^{3}}\left(Z^{b} v \cdot \nabla \partial_{t} Z^{c} d\right) \cdot \partial_{t} Z^{a} d \mathrm{~d} x-\sum_{b+c=a} C_{a}^{b} \int_{\mathbb{R}^{3}}\left(Z^{b} v \cdot \nabla Z^{c} d\right) \cdot \partial_{t}^{2} Z^{a} d \mathrm{~d} x
$$

Thus $M_{1}$ can be bounded by

$$
-\sum_{b+c=a} C_{a}^{b} \partial_{t} \int_{\mathbb{R}^{3}}\left(Z^{b} v \cdot \nabla Z^{c} d\right) \cdot \partial_{t} Z^{a} d \mathrm{~d} x+\int_{\mathbb{R}^{3}}\left|Z^{|a|} v\right|\left|\partial Z^{|a|} d\right|\left|\partial^{2} Z^{|a|} d\right| \mathrm{d} x .
$$

Now we estimate the last term in the above expression. For the integral domain of $\{r \leq\langle t\rangle / 2\}$, one deduces from Lemma 3.2 and the Sololev inequality $\|u\|_{L^{\infty}} \lesssim\|\nabla u\|_{L^{2}}^{\frac{1}{2}}\left\|\nabla^{2} u\right\|_{L^{2}}^{\frac{1}{2}}$ that

$$
\begin{aligned}
& \int_{r \leq\langle t\rangle / 2}\left|Z^{|a|} v\left\|\partial Z^{|a|} d\right\| \partial^{2} Z^{|a|} d\right| \mathrm{d} x \\
& \lesssim\langle t\rangle^{-1}\left\|Z^{|a|} v\right\|_{L^{\infty}}\left\|\partial Z^{|a|} d\right\|_{L^{2}}\left\|\langle t-r\rangle \partial^{2} Z^{|a|} d\right\|_{L^{2}(r \leq\langle t\rangle / 2)} \\
& \lesssim\langle t\rangle^{-1}\left\|\nabla Z^{\kappa-1} v\right\|_{L^{2}}^{\frac{1}{2}}\left(E_{\kappa}^{v}\right)^{\frac{1}{4}}\left(E_{\kappa-1}^{d} E_{\kappa+1}^{d}\right)^{\frac{1}{2}} .
\end{aligned}
$$

For the integral region of $\{r \geq\langle t\rangle / 2\}$, we have from (2.18) that

$$
\begin{aligned}
& \int_{r \geq\langle t\rangle / 2}\left|Z ^ { | a | } v \left\|\left|\partial Z^{|a|} d \| \partial^{2} Z^{|a|} d\right| \mathrm{d} x\right.\right. \\
& \lesssim\langle t\rangle^{-1}\left\|r Z^{|a|} v\right\|_{L^{\infty}(r \geq\langle t\rangle / 2)}\left\|\partial Z^{|a|} d\right\|_{L^{2}}\left\|\partial^{2} Z^{|a|} d\right\|_{L^{2}} \\
& \lesssim\langle t\rangle^{-1}\left\|\nabla Z^{\kappa} v\right\|_{L^{2}}^{\frac{1}{2}}\left(E_{\kappa}^{v}\right)^{\frac{1}{4}}\left(E_{\kappa-1}^{d} E_{\kappa+1}^{d}\right)^{\frac{1}{2}} .
\end{aligned}
$$

Next, we write $M_{2}$ as:

$$
M_{2}=\int_{\mathbb{R}^{3}} \sum_{b+c+e=a} C_{a}^{b, c}\left(\nabla Z^{b} d \cdot \nabla Z^{c} d-\partial_{t} Z^{b} d \cdot \partial_{t} Z^{c} d\right) Z^{e} d \cdot \partial_{t} Z^{a} d \mathrm{~d} x .
$$

In the integral domain of $\{r \leq\langle t\rangle / 2\}$, employing (2.20), (2.21) and Lemma 3.2 yields

$$
\begin{aligned}
& \int_{r \leq\langle t\rangle / 2} \sum_{\substack{b+c+e=a \\
|e| \geq|a| / 2}} C_{a}^{b, c}\left(\nabla Z^{b} d \cdot \nabla Z^{c} d-\partial_{t} Z^{b} d \cdot \partial_{t} Z^{c} d\right) Z^{e} d \cdot \partial_{t} Z^{a} d \mathrm{~d} x \\
& \lesssim \sum_{|e| \geq|a| / 2}\left\|\partial Z^{[|a| / 2]} d\right\|_{L^{6}(r \leq\langle t\rangle / 2)}\left\|\partial Z^{[|a| / 2]} d\right\|_{L^{6}(r \leq\langle t\rangle / 2)}\left\|Z^{e} d\right\|_{L^{6}}\left\|\partial_{t} Z^{a} d\right\|_{L^{2}} \\
& +\sum_{|e| \leq \frac{|a|}{2}}\left\|\partial Z^{|a|} d\right\|_{L^{6}(r \leq\langle t\rangle / 2)}\left\|\partial Z^{[|a| / 2]} d\right\|_{L^{3}(r \leq\langle t\rangle / 2)}\left\|Z^{e} d\right\|_{L^{\infty}}\left\|\partial_{t} Z^{a} d\right\|_{L^{2}} \\
& \lesssim\langle t\rangle^{-\frac{3}{2}} E_{\kappa-1}^{d}\left(E_{\kappa+1}^{d}\right)^{\frac{1}{2}}\left(1+\left(E_{\kappa-1}^{d}\right)^{\frac{1}{2}}\right) .
\end{aligned}
$$

To estimate the integral domain of $\{r \geq\langle t\rangle / 2\}$, we need to use the null condition. To this end, we first write

$$
\begin{aligned}
& \sum_{b+c+e=a} C_{a}^{b, c} \int_{r \geq\langle t\rangle / 2}\left(\nabla Z^{b} d \cdot \nabla Z^{c} d-\partial_{t} Z^{b} d \cdot \partial_{t} Z^{c} d\right) Z^{e} d \cdot \partial_{t} Z^{a} d \mathrm{~d} x \\
= & \sum_{b+c+e=a} C_{a}^{b, c} \int_{r \geq\langle t\rangle / 2}\left(\omega_{i} \partial_{t}+\nabla_{i}\right) Z^{b} d \cdot\left(\omega_{i} \partial_{t}-\nabla_{i}\right) Z^{c} d Z^{e} d \cdot \partial_{t} Z^{a} d \mathrm{~d} x \\
\lesssim & \sum_{b+c+e=a} \int_{r \geq\langle t\rangle / 2}\left|\left(\omega_{i} \partial_{t}+\nabla_{i}\right) Z^{b} d\left\|\partial Z^{c} d\right\| Z^{e} d \| \partial_{t} Z^{a} d\right| \mathrm{d} x,
\end{aligned}
$$


where $\omega=x / r$. Consequently, employing (2.17) and Lemma 3.3. the above can be further controlled by

$$
\begin{aligned}
& \sum_{|e| \leq|a| / 2}\left\|\left(\omega_{i} \partial_{t}+\nabla_{i}\right) Z^{|a|} d\right\|_{L^{\infty}(r \geq\langle t\rangle / 2)}\left\|\partial Z^{|a|} d\right\|_{L^{2}}\left\|Z^{e} d\right\|_{L^{\infty}}\left\|\partial_{t} Z^{a} d\right\|_{L^{2}} \\
& +\sum_{|e| \geq|a| / 2}\left\|\left(\omega_{i} \partial_{t}+\nabla_{i}\right) Z^{[|a| / 2]} d\right\|_{L^{\infty}(r \geq\langle t\rangle / 2)}\left\|\partial Z^{[|a| / 2]} d\right\|_{L^{3}}\left\|Z^{e} d\right\|_{L^{6}}\left\|\partial_{t} Z^{a} d\right\|_{L^{2}} \\
& \lesssim\langle t\rangle^{-\frac{3}{2}}\left(E_{\kappa+1}^{d}\right)^{\frac{1}{2}} E_{\kappa-1}^{d}\left(1+E_{\kappa-1}^{d}\right)^{\frac{1}{2}} .
\end{aligned}
$$

Here we have used the spatial decomposition along radial and reverse direction:

$$
\nabla=\frac{x}{r} \partial_{r}-\frac{\omega}{r} \wedge \Omega
$$

Now we estimate $M_{3}, M_{4}$ and $M_{5}$ which contain cubic, quartic and quintic terms but no quadratic term. Thanks to (2.17), (2.19) and Lemma 3.2, one gets

$$
\begin{aligned}
M_{3}= & -\sum_{b+c+e=a} C_{a}^{b, c} \int_{\mathbb{R}^{3}} Z^{b} v \cdot \nabla\left(Z^{c} v \cdot \nabla Z^{e} d\right) \cdot \partial_{t} Z^{a} d \mathrm{~d} x \\
\lesssim & \left(\int_{r \geq\langle t\rangle / 2}+\int_{r \geq\langle t\rangle / 2}\right)\left|Z^{|a|} v\left\|Z^{|a|+1} v\right\| \nabla Z^{|a|+1} d \| \partial_{t} Z^{a} d\right| \mathrm{d} x \\
\leq & \left\|Z^{|a|} v\right\|_{L^{\infty}(r \geq\langle t\rangle / 2)}\left\|Z^{|a|+1} v\right\|_{L^{\infty}}\left\|\nabla Z^{|a|+1} d\right\|_{L^{2}}\left\|\partial_{t} Z^{a} d\right\|_{L^{2}} \\
& +\left\|Z^{|a|+1} v\right\|_{L^{6}}^{2}\left\|\nabla Z^{|a|+1} d\right\|_{L^{6}(r \leq\langle t\rangle / 2)}\left\|\partial_{t} Z^{a} d\right\|_{L^{2}} \\
\leq & \langle t\rangle^{-1}\left\|\nabla Z^{\kappa} v\right\|_{L^{2}}^{\frac{1}{2}}\left(E_{\kappa}^{v}\right)^{\frac{3}{2}}\left(E_{\kappa+1}^{d} E_{\kappa-1}^{d}\right)^{\frac{1}{2}}
\end{aligned}
$$

Moreover, we have

$$
\begin{aligned}
M_{4} & =-\sum_{b+c+e+f=a} C_{a}^{b, c, e} \int_{\mathbb{R}^{3}}\left[2\left(Z^{b} v \cdot \nabla\right) Z^{c} d \cdot \partial_{t} Z^{e} d\right] Z^{f} d \cdot \partial_{t} Z^{a} d \mathrm{~d} x \\
& \lesssim \sum_{b+c+e+f=a} \int_{\mathbb{R}^{3}}\left|Z^{b} v\left\|\partial Z^{c} d\right\| \partial Z^{e} d\left\|Z^{f} d\right\| \partial_{t} Z^{a} d\right| \mathrm{d} x .
\end{aligned}
$$

By Sobolev inequalities and Lemma 3.4, the above can be further bounded by

$$
\begin{aligned}
& \sum_{\frac{|a|}{2} \leq|f| \leq|a|}\left\|Z^{|a|} v\right\|_{L^{6}}\left\|\partial Z^{|a|} d\right\|_{L^{6}}\left\|\partial Z^{[|a| / 2]} d\right\|_{L^{\infty}}\left\|Z^{f} d\right\|_{L^{6}}\left\|\partial_{t} Z^{a} d\right\|_{L^{2}} \\
& +\sum_{|f| \leq \frac{|a|}{2}}\left\|Z^{|a|} v\right\|_{L^{\infty}}\left\|\partial Z^{|a|} d\right\|_{L^{2}}\left\|\partial Z^{[|a| / 2]} d\right\|_{L^{\infty}}\left\|Z^{f} d\right\|_{L^{\infty}}\left\|\partial_{t} Z^{a} d\right\|_{L^{2}} \\
& \lesssim\langle t\rangle^{-1}\left\|\nabla Z^{\kappa} v\right\|_{L^{2}}^{\frac{1}{2}}\left\|\nabla Z^{\kappa-1} v\right\|_{L^{2}}^{\frac{1}{2}}\left(E_{\kappa-1}^{d}\right)^{\frac{3}{2}}\left[\left(E_{\kappa+1}^{d}\right)^{\frac{1}{2}}+1\right] .
\end{aligned}
$$

The term $M_{5}$ can be estimated as

$$
\begin{aligned}
M_{5} & =-\sum_{b+c+e+f+g=a} C_{a}^{b, c, e, f} \int_{\mathbb{R}^{3}}\left[\left(Z^{b} v \cdot \nabla\right) Z^{c} d \cdot\left(\left(Z^{e} v \cdot \nabla\right) Z^{f} d\right)\right] Z^{g} d \cdot \partial_{t} Z^{a} d \mathrm{~d} x \\
& \lesssim \sum_{|e|+|f|+|g| \leq|a|} \int_{\mathbb{R}^{3}}\left|Z^{|a|} v\right|^{2}\left|\nabla Z^{e} d\right|\left|\nabla Z^{f} d\left\|Z^{g} d\right\| \partial_{t} Z^{a} d\right| \mathrm{d} x \\
& \lesssim \sum_{|g| \leq \frac{|a|}{2}}\left\|Z^{|a|} v\right\|_{L^{6}}^{2}\left\|\partial Z^{|a|} d\right\|_{L^{2}}\left\|\partial Z^{[|a| / 2]} d\right\|_{L^{6}}\left\|Z^{g} d\right\|_{L^{\infty}}\left\|\partial_{t} Z^{a} d\right\|_{L^{2}}
\end{aligned}
$$




$$
\begin{aligned}
& \quad+\sum_{\frac{|a|}{2} \leq|g| \leq|a|}\left\|Z^{|a|} v\right\|_{L^{6}}^{2}\left\|\partial Z^{|a|} d\right\|_{L^{2}}\left\|\partial Z^{[|a| / 2]} d\right\|_{L^{\infty}}\left\|Z^{g} d\right\|_{L^{6}}\left\|\partial_{t} Z^{a} d\right\|_{L^{2}} . \\
& \lesssim\left\|\nabla Z^{\kappa} v\right\|_{L^{2}}^{2}\left(E_{\kappa-1}^{d}\right)^{\frac{3}{2}}\left(1+E_{\kappa-1}^{d}\right)^{\frac{1}{2}} .
\end{aligned}
$$

Combining all the estimates of $M_{1}$ to $M_{5}$ leads

$$
\begin{aligned}
& \frac{d}{d t} \int_{\mathbb{R}^{n}}\left[\frac{1}{2}\left|\partial Z^{a} d\right|^{2}+\sum_{b+c=a} C_{a}^{b}\left(Z^{b} v \cdot \nabla Z^{c} d\right) \cdot \partial_{t} Z^{a} d\right] \mathrm{d} x \\
& \lesssim\langle t\rangle^{-1}\left\|\nabla Z^{\kappa} v\right\|_{L^{2}}^{\frac{1}{2}}\left(E_{\kappa}^{v}\right)^{\frac{1}{4}}\left(E_{\kappa-1}^{d} E_{\kappa+1}^{d}\right)^{\frac{1}{2}}+\langle t\rangle^{-\frac{3}{2}} E_{\kappa-1}^{d}\left(E_{\kappa+1}^{d}\right)^{\frac{1}{2}}+\left\|\nabla Z^{\kappa} v\right\|_{L^{2}}^{2} E_{\kappa-1}^{d} .
\end{aligned}
$$

Summing over $|a| \leq \kappa-2$, and noting that

$$
\begin{aligned}
& \sum_{|a| \leq \kappa-2} \int_{\mathbb{R}^{n}}\left[\frac{1}{2}\left|\partial Z^{a} d\right|^{2}+\sum_{b+c=a} C_{a}^{b}\left(Z^{b} v \cdot \nabla Z^{c} d\right) \cdot \partial_{t} Z^{a} d\right] \mathrm{d} x \\
& \sim \sum_{|a| \leq \kappa-2} \frac{1}{2} \int_{\mathbb{R}^{n}}\left|\partial Z^{a} d\right|^{2} \mathrm{~d} x=\frac{1}{2} E_{\kappa-1}^{d} .
\end{aligned}
$$

Thus we finish the proof of (2.11).

\section{Acknowledgement.}

The authors would like to thank Professor Fanghua Lin for suggesting this problem and his helpful discussion. We are also grateful to the hospitality of Courant Institute of Mathematical Sciences where this work was carried out. Y. Cai was sponsored by the China Scholarship Council (No. 201606100111) for one year at New York University, Courant Institute of Mathematics Sciences. W. Wang is supported by NSF of China under Grant No. 11922118, 11931010 and 11771388, and the Young Elite Scientists Sponsorship Program by CAST.

\section{References}

[1] Alinhac, S. The null condition for quasilinear wave equations in two space dimensions I. Invent. Math. 145 (2001), no. 3, 597-618.

[2] Bressan, A., Zheng, Y. Conservative solutions to a nonlinear variational wave equation. Comm. Math. Phys. 266 (2006), no. 2, 471-497.

[3] Cai, Y., Lei, Z., Lin, F. H. and Masmoudi, N. Vanishing Viscosity Limit for the Incompressible Viscoelasticity in Two Dimensions. Preprint on Comm. Pure Appl. Math. arXiv:1703.01176.

[4] Ericksen, J., Conservation laws for liquid crystals. Trans. Soc. Rheol. 5, 22-34 (1961)

[5] Ericksen, J., Liquid crystals with variable degree of orientation, Arch. Ration. Mech. Anal., 113 (1991), 97-120.

[6] Hong, M.-C. Global existence of solutions of the simplified Ericksen-Leslie system in dimension two. Calc. Var. Partial Diff. Equ. 40 (2011), 15-36.

[7] Hong M.-C., Xin Z. Global existence of solutions of the liquid crystal flow for the Oseen-Frank model in $\mathbb{R}^{2}$. Adv. Math. 231 (2012), 1364-1400.

[8] Huang J., Lin FH, Wang CY. Regularity and existence of global solutions to the Ericksen-Leslie system in $\mathbb{R}^{2}$. Commun. Math. Phys. 331 (2014), 805-850. 
[9] Huang T, Wang CY. Blow up criterion for nematic liquid crystal flows. Commun. Partial Diff. Equ. 37(2012), 875-884.

[10] Jiang, N. and Luo, Y.On well-posedness of Ericksen-Leslie's hyperbolic incompressible liquid crystal model, SIAM J. Math. Anal. 51 (2019), no. 1, 403-434.

[11] Kessenich, P. Global existence with small initial data for three-dimensional incompressible isotropic viscoelastic materials, available online at arXiv:0903.2824

[12] Klainerman, S. and Machedon M., Space-time estimates for null forms and the local existence theorem, Comm. Pure Appl. Math. 46 (1993), no. 9, 1221-1268.

[13] Klainerman, S. and Machedon M., Smoothing estimates for null forms and applications, Duke Math. J. 81 (1995), no. 1, 99-133 (1996).

[14] Klainerman, S. and Machedon M., On the regularity properties of a model problem related to wave maps, Duke Math. J. 87 (1997), no. 3, 553-589.

[15] Klainerman, S., Selberg, S. Remark on the optimal regularity for equations of the wave maps type. Comm. Partial Differential Equations 22 (1997), no. 5-6, 901-918.

[16] Klainerman, S., Selberg, S. Bilinear estimates and applications to nonlinear wave equations. Commun. Contemp. Math. 4 (2002), no. 2, 223-295.

[17] Klainerman, S. and Sideris, T. C. On almost global existence for nonrelativistic wave equations in 3D. Comm. Pure Appl. Math. 49 (1996), 307-321.

[18] Klainerman, S., Rodnianski, I. On the global regularity of wave maps in the critical Sobolev norm. Internat. Math. Res. Notices 2001, no. 13, 655-677.

[19] Krieger, J. Null-form estimates and nonlinear waves. Adv. Differential Equations 8 (2003), no. $10,1193-1236$.

[20] Krieger, J. Global regularity of wave maps from $R^{3+1}$ to surfaces. Comm.Math. Phys. 238 (2003), no. $1-2,333-366$.

[21] Krieger, J. Global regularity of wave maps from $R^{2+1}$ to $H^{2}$. Small energy. Comm. Math. Phys. 250 (2004), no. 3, 507-580.

[22] Krieger, J.; Schlag, W. Concentration compactness for critical wave maps. EMS Monographs in Mathematics. European Mathematical Society, Zürich, 2012.

[23] Krieger, J., Schlag, W., Tataru, D. Renormalization and blow up for charge one equivariant critical wave maps. Invent. Math. 171 (2008), no. 3, 543-615.

[24] Lei, Z. Global well-posedness of incompressible elastodynamics in two dimensions. Comm. Pure Appl. Math. 69 (2016), 2072-2106.

[25] Lei, Z., Li, D., Zhang, X.Y. Remarks of global wellposedness of liquid crystal flows and heat flows of harmonic maps in two dimensions. Proc. Amer. Math. Soc. 142 (2014), no. 11, 3801-3810.

[26] Lei, Z., Wang, F. Uniform bound of the highest energy for the three dimensional incompressible elastodynamics. Arch. Ration. Mech. Anal. 216 (2015), no. 2, 593-622.

[27] Lemarie-Rieusset, P. G. Recent Developments in the Navier-Stokes Problem. Boca Ration,FL: Chapman \& Hall/CRC, 2002.

[28] Leslie, F.M. Some constitutive equations for liquid crystals. Arch.Ration. Mech.Anal. 28, 265-283 (1968) 
[29] Li J-K., Titi E. and Xin Z-P., On the uniqueness of weak solutions to the Ericksen-Leslie liquid crystal model in $\mathbb{R}^{2}$. Math. Models Methods Appl. Sci. 26 (2016), no. 4, 803-822.

[30] Li XL, Wang DH. Global solution to the incompressible flow of liquid crystals. J. Diff. Equ. $252(2012), 745-767$.

[31] Lin, F.-H. Nonlinear theory of defects in nematic liquid crystals; phase transition and flow phenomena. Comm. Pure Appl. Math. 42 (1989), no. 6, 789-814.

[32] Lin F.-H., Lin J.-Y., Wang C.-Y., Liquid crystal flows in two dimensions. Arch. Ration. Mech. Anal. 197 (2010), no. 1, 297-336.

[33] Lin F.-H. and Liu C., Nonparabolic dissipative systems modeling the flow of liquid crystals. Comm. Pure Appl. Math. 48 (1995), no. 5, 501-537.

[34] Lin F.-H. and Liu C., Partial regularity of the dynamic system modeling the flow of liquid crystals. Discrete Contin. Dynam. Systems 2 (1996), no. 1, 1-22.

[35] Lin F.-H. and Liu C., Existence of solutions for the Ericksen-Leslie system. Arch. Ration. Mech. Anal. 154 (2000), no. 2, 135-156.

[36] Lin F.-H.,Wang C. On the uniqueness of heat flow of harmonic maps and hydrodynamic flow of nematic liquid crystals. Chin. Ann. Math. Ser. B 31(2010), 921-938.

[37] Lin F. H.,Wang C. Global existence of weak solutions of the nematic liquid crystal flow in dimensions three. Comm. Pure Appl. Math. 69 (2016), no. 8, 1532-1571.

[38] Nahmod, A., Stefanov, A., Uhlenbeck, K. On the well-posedness of the wave map problem in high dimensions. Comm. Anal. Geom. 11 (2003), no. 1, 49-83.

[39] Rodnianski, I. and Sterbenz, J. On the Formation of Singularities in the Critical O(3) SigmaModel. Ann. of Math. (2) 172 (2010), no. 1, 187-242.

[40] Shatah J., Weak solutions and development of singularities in the SU(2)- $\sigma$ model, Comm. Pure Appl. Math. 41 (1988) 459-469.

[41] Sideris, T. C. Nonresonance and global existence of prestressed nonlinear elastic waves. Ann. of Math. (2) 151 (2000), no. 2, 849-874.

[42] Sterbenz J., Tataru D., Energy dispersed large data wave maps in 2+1 dimensions, Comm. Math. Phys. 298 (2010), no. 1, 139-230.

[43] Sterbenz J., Tataru D., Regularity of wave-maps in dimension 2+1, Comm. Math. Phys. 298 (2010), no. 1, 231-264.

[44] Struwe, M. Equivariant wave maps in two space dimensions. Comm. Pure Appl.Math. 56 (2003), no. $7,815-823$.

[45] Tao T., Global regularity of wave maps I. Small critical Sobolev norm in high dimension. IMRN, 7, 299-328 (2001).

[46] Tao T., Global Regularity of Wave Maps II. Small Energy in Two Dimensions, Commun. Math. Phys. 224, 443-544 (2001)

[47] Tao T., Global regularity of wave maps III. Large energy from $R^{1+2}$ to hyperbolic spaces, arXiv:0805.4666

[48] Tao T., Global regularity of wave maps IV. Absence of stationary or self-similar solutions in the energy class, http://arxiv.org/abs/0806.3592v2[math.AP], 2009 
[49] Tao T., Global regularity of wave maps V. Large data local well-posedness in the energy class, http://arxiv.org/abs/0808.0368v2[math.AP], 2009

[50] Tao T., Global regulrity of wave maps VI. Minimal energy blowup solutions, http://arxiv.org/abs/0906.2833v2[math.AP], 2009

[51] Tao T., Global regularity of wave maps VII. Control of delocalised or dispersed solutions , https://arxiv.org/abs/0908.0776v2[math.AP], 2009

[52] Tataru D., Local and global results for wave maps I, Comm.PDE 23, 1781-1793 (1998).

[53] Tataru D., On global existence and scattering for the wave maps equation, Amer. J. Math. 123 (2001), no. 1, 37-77.

[54] Tataru D., Rough solutions for the wave maps equation, Amer. J. Math. 127 (2005), no. 2, 293377.

[55] Tataru, D. The wave maps equation. Bull. Amer. Math. Soc. 41 (2004), no. 2, $185-204$.

[56] Wang, M. and Wang, W. Global existence of weak solution for the 2-D Ericksen-Leslie system. Calc. Var. Partial Differential Equations 51 (2014), no. 3-4, 915-962.

[57] Wang, M., Wang, W.D., Zhang, Z.F. On the uniqueness of weak solutions for the 2-D EricksenLeslie system. Discrete Contin. Dyn. Syst. Ser. B 21(2016), 919-941.

[58] Wang, W., Zhang, P. and Zhang, Z. Well-posedness of the Ericksen-Leslie system. Arch. Ration. Mech. Anal. 210 (2013), no. 3, 837-855.

[59] Xu X., Zhang Z.F. Global regularity and uniqueness of weak solution for the 2-D liquid crystal flows. J. Diff. Equ. 252(2012), 1169-1181.

[60] Zhang P. and Zheng Y-X., Weak solutions to a nonlinear variational wave equation with general data. Ann. Inst. H. Poincaré Anal. Non Linéaire 22 (2005), no. 2, 207-226.

[61] Zhang P. and Zheng Y-X., Conservative solutions to a system of variational wave equations of nematic liquid crystals. Arch. Ration. Mech. Anal. 195 (2010), no. 3, 701-727.

[62] Zhang P. and Zheng Y-X., Energy conservative solutions to a one-dimensional full variational wave system. Comm. Pure Appl. Math. 65 (2012), no. 5, 683-726. 